\title{
NeuroImage
}

\section{Mapping cortical change in Alzheimer's disease, brain development, and schizophrenia}

\author{
Paul M. Thompson, ${ }^{\mathrm{a}, *}$ Kiralee M. Hayashi, ${ }^{\mathrm{a}}$ Elizabeth R. Sowell, ${ }^{\mathrm{a}}$ Nitin Gogtay, ${ }_{\mathrm{c}}^{\mathrm{b}}$ Jay N. Giedd, ${ }^{\mathrm{b}}$ \\ Judith L. Rapoport, ${ }^{\mathrm{b}}$ Greig I. de Zubicaray, ${ }^{\mathrm{c}}$ Andrew L. Janke, ${ }^{\mathrm{c}}$ Stephen E. Rose, ${ }^{\mathrm{c}}$ James Semple, ${ }^{\mathrm{d}}$ \\ David M. Doddrell, ${ }^{\mathrm{c}}$ Yalin Wang, ${ }^{\mathrm{e}}$ Theo G.M. van Erp, ${ }^{\mathrm{f}}$ Tyrone D. Cannon, ${ }^{\mathrm{f}}$ and Arthur W. Toga ${ }^{\mathrm{a}}$ \\ ${ }^{a}$ Laboratory of Neuro Imaging, Brain Mapping Division, Department of Neurology, UCLA School of Medicine, Los Angeles, CA 90095-1769, United States \\ ${ }^{\mathrm{b}}$ Child Psychiatry Branch, NIMH, Bethesda, MD 20892, United States \\ ${ }^{\circ}$ Centre for Magnetic Resonance, University of Queensland, Brisbane, QLD 4072, Australia \\ ${ }^{\mathrm{d}}$ GlaxoSmithKline Pharmaceuticals plc, Addenbrooke's Centre for Clinical Investigation, Addenbrooke's Hospital, Cambridge, UK \\ ${ }^{\mathrm{e}}$ UCLA Department of Mathematics, Los Angeles, CA 90095-1555, United States

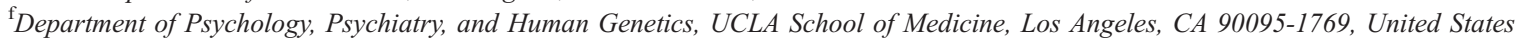

Available online 25 September 2004

This paper describes algorithms that can identify patterns of brain structure and function associated with Alzheimer's disease, schizophrenia, normal aging, and abnormal brain development based on imaging data collected in large human populations. Extraordinary information can be discovered with these techniques: dynamic brain maps reveal how the brain grows in childhood, how it changes in disease, and how it responds to medication. Genetic brain maps can reveal genetic influences on brain structure, shedding light on the nature-nurture debate, and the mechanisms underlying inherited neurobehavioral disorders. Recently, we created time-lapse movies of brain structure for a variety of diseases. These identify complex, shifting patterns of brain structural deficits, revealing where, and at what rate, the path of brain deterioration in illness deviates from normal. Statistical criteria can then identify situations in which these changes are abnormally accelerated, or when medication or other interventions slow them. In this paper, we focus on describing our approaches to map structural changes in the cortex. These methods have already been used to reveal the profile of brain anomalies in studies of dementia, epilepsy, depression, childhoodand adult-onset schizophrenia, bipolar disorder, attention-deficit/ hyperactivity disorder, fetal alcohol syndrome, Tourette syndrome, Williams syndrome, and in methamphetamine abusers. Specifically, we describe an image analysis pipeline known as cortical pattern matching that helps compare and pool cortical data over time and across subjects. Statistics are then defined to identify brain structural differences between groups, including localized alterations in cortical thickness, gray matter density (GMD), and asymmetries in cortical organization. Subtle features, not seen in individual brain

* Corresponding author. Reed Neurological Research Center, Laboratory of Neuro Imaging, Brain Mapping Division, Department of Neurology, UCLA School of Medicine, Room 4238, 710 Westwood Plaza, Los Angeles, CA 90095-1769. Fax: +1 3102065518.

E-mail address: thompson@loni.ucla.edu (P. Thompson).

Available online on ScienceDirect (www.sciencedirect.com.) scans, often emerge when population-based brain data are averaged in this way. Illustrative examples are presented to show the profound effects of development and various diseases on the human cortex. Dynamically spreading waves of gray matter loss are tracked in dementia and schizophrenia, and these sequences are related to normally occurring changes in healthy subjects of various ages.

(C) 2004 Published by Elsevier Inc.

Keywords: Alzheimer's disease; Brain development; Schizophrenia

\section{Introduction}

Brain imaging continues to provide new and remarkable insights on how disease impacts the human brain. Large-scale brain mapping initiatives are charting brain structure and function in hundreds or even thousands of human subjects across the life span (e.g., Good et al., 2001, $N=465$; Mazziotta et al., 2001; $N=$ 7000). The individuals surveyed include twin populations and patients with Alzheimer's disease, schizophrenia, and other neurological and psychiatric disorders. At the cutting edge of this research are mathematical and computational strategies to compare and contrast imaging information from large populations, and to map disease effects on the brain. Such techniques are now revealing dynamic waves of brain change in development, dementia, and psychosis. Mathematical models are also identifying how drug treatments, risk genes, and demographic factors modulate these dynamic processes. Another related type of brain map - a genetic brain map - can also reveal how heredity and environmental factors influence cortical development and disease (Cannon et al., 2002; Thompson et al., 2001a,b). These brain 
mapping techniques empower disease detection, exploration, and intervention, and offer new insights in clinical trials assessing drugs that slow degenerative brain changes (Ashburner et al., 2003; Jack et al., 2003; Zijdenbos et al., 1996).

Many brain imaging studies focus on the cerebral cortex, which changes profoundly during development and disease. Nonetheless, cortical geometry is complex and varies widely from individual to individual. This presents a challenge for all brain mapping efforts that aim to pool neuroimaging data across subjects. Unless mathematical tactics are developed to model the structural and functional variation of the human brain, efforts to detect group differences in brain structure are limited considerably, and disease effects on cortical anatomy are difficult to identify.

\section{Computational anatomy}

As imaging studies expand into ever-larger populations, we and others have developed techniques that have mapped unsuspected patterns of brain changes in childhood (Giedd et al., 1999; Gogtay et al., 2004; Paus et al., 1999; Sowell et al., 2001, 2002, in press,b; Thompson et al., 2000a,b) and dynamic waves of tissue loss in dementia and schizophrenia (Rapoport et al., 1999; Thompson et al., 2001a,b, 2003). Maps of disease effects on the brain, computed from serial magnetic resonance imaging (MRI) scans of patients (Janke et al., 2001) or those at genetic risk (Cannon et al., 2002), can clarify disease progression and transmission, and can also provide therapeutic targets (cf. Fox et al., 2001). All these efforts draw on methods from the rapidly growing field of computational anatomy (see e.g., Ashburner et al., 2003; Bankman, 1999: Bookstein, 2001: Chung, 2001: Csernansky et al., 1998; Davatzikos, 1996, 2001; Drury and Van Essen, 1997; Evans et al., 1994; Fischl and Dale, 2000; Fitzpatrick and Sonka, 2000; Gee and Bajcsy, 1998; Gerig et al., 2001; Grenander and Miller, 1998; Leahy and Insana, 2001; Miller et al., 2002; Sereno et al., 1996; Thompson and Toga, 2003a,b; Thompson et al., 2000a,b, 2001a,b, cf. Toga, 1998; Toga and Mazziotta, 2002; see other papers in this issue for recent developments and reviews).

The methods described in this paper represent one set of approaches used in computational anatomy today. Computational anatomy uses mathematical techniques from differential geometry, numerical analysis, and the theory of partial differential equations (Sapiro, 2001) to model objects and processes in brain images. Geometrical surfaces, for example, are often used to represent the shape of brain structures such as the cerebral cortex (Fischl et al., 2001; Thompson and Toga, 1996; van Essen, in press). Techniques to analyze and compare cortical structure have advanced through many years of research in computer vision, artificial intelligence, image analysis, and computer graphics. Neuroscience studies applying these methods typically aim to uncover patterns of altered structure and function in healthy and diseased populations using novel mathematics to identify new features, to compare brain measures, or to increase the sensitivity to detect statistically significant differences. Detecting systematic effects of disease on brain structure is challenging as it requires: (1) computational techniques to generate average patterns of brain structure in human populations (see Fig. 1); (2) statistical methods that work with scalar or vector fields to encode individual variations in brain structure and identify significant group differences or changes over time (Figs. 2 and 3); and (3) large and richly characterized image databases, with related cognitive, clinical, demographic, and often genetic, data on patients and healthy controls.

\section{Averaging brain structure}

One of the most fundamental challenges in brain mapping is how to average and compare brain structure across subjects. The anatomy of different subjects varies widely, especially the gyral patterns of the cortex, and this presents a problem when averaging brain images together, as can be seen in an example (Fig. 1).

Many diseases affect cortical anatomy, but there have been major difficulties in developing average and statistical representations of the effects of disease on the cortex, given the extreme variations in cortical patterning across subjects. One solution to this, proposed here, is to build explicit geometric models of the cortex using parametric surfaces and to build deformation maps on the geometric models that explicitly associate corresponding cortical regions across subjects. A similar 'surface-based' approach has also been taken by other groups developing frameworks to visualize or analyze cortical data (e.g., Hurdal and Stephenson, in press; Mangin et al., in press; Tosun et al., in press; van Essen, in press, this volume).

A clear benefit of this approach is that it can be used to create average models of cortical anatomy that retain anatomic features (e.g., sulcal landmarks) found consistently across subjects. Templates of cortical anatomy can be built retaining detailed information about individual variability. Functional or structural data from many subjects can then be transferred to a common neuroanatomical template while adjusting for individual differences in gyral patterning. Features that have a consistent relation to the gyral anatomy are then greatly reinforced in the group average models (Fig. 1). The explicit matching of anatomy eliminates much of the confounding anatomical variance when pooling data across subjects and makes consistent functional and structural patterns easier to identify (Rasser et al., in press; Thompson et al., 2001a,b).

When matching cortical anatomy between subjects, mathematical criteria can be applied to enforce the matching of key functional or anatomic landmarks from one data set to another. 3D deformation maps can be computed to match these features exactly, while deforming one surface onto another. These deformation algorithms often draw on methods from continuum mechanics, extending concepts to brain images that were originally developed to model the deformation of $3 \mathrm{D}$ elastic and fluid media (these tools are described later).

A second benefit of surface-based cortical modeling is that the anatomical variability of the cortex can be studied by constructing mappings that deform one cortex onto another. If these deformation mappings are analyzed statistically, group differences in cortical anatomy, or hemispheric asymmetries, can be pinpointed and mapped. Their anatomical profile can also be visualized. Regions with significant differences in cortical thickness, gyral patterning, or other cortical attributes can be visualized in color on a graphically rendered anatomical surface. Systematic differences or changes in cortical organization, gray matter distribution, cortical thickness, or asymmetry can then be distinguished from normal variations, and statistical criteria can be developed to assess if cortical anatomy is abnormal by referring to normative data on anatomical variation (Thompson et al., 1997).

\section{Overview of paper}

This paper gives an overview of methods we have developed to analyze cortical anatomy. Illustrative data from various neuroscience projects are presented, as well as the mathematics used to compute them. We describe the types of maps and models that can 


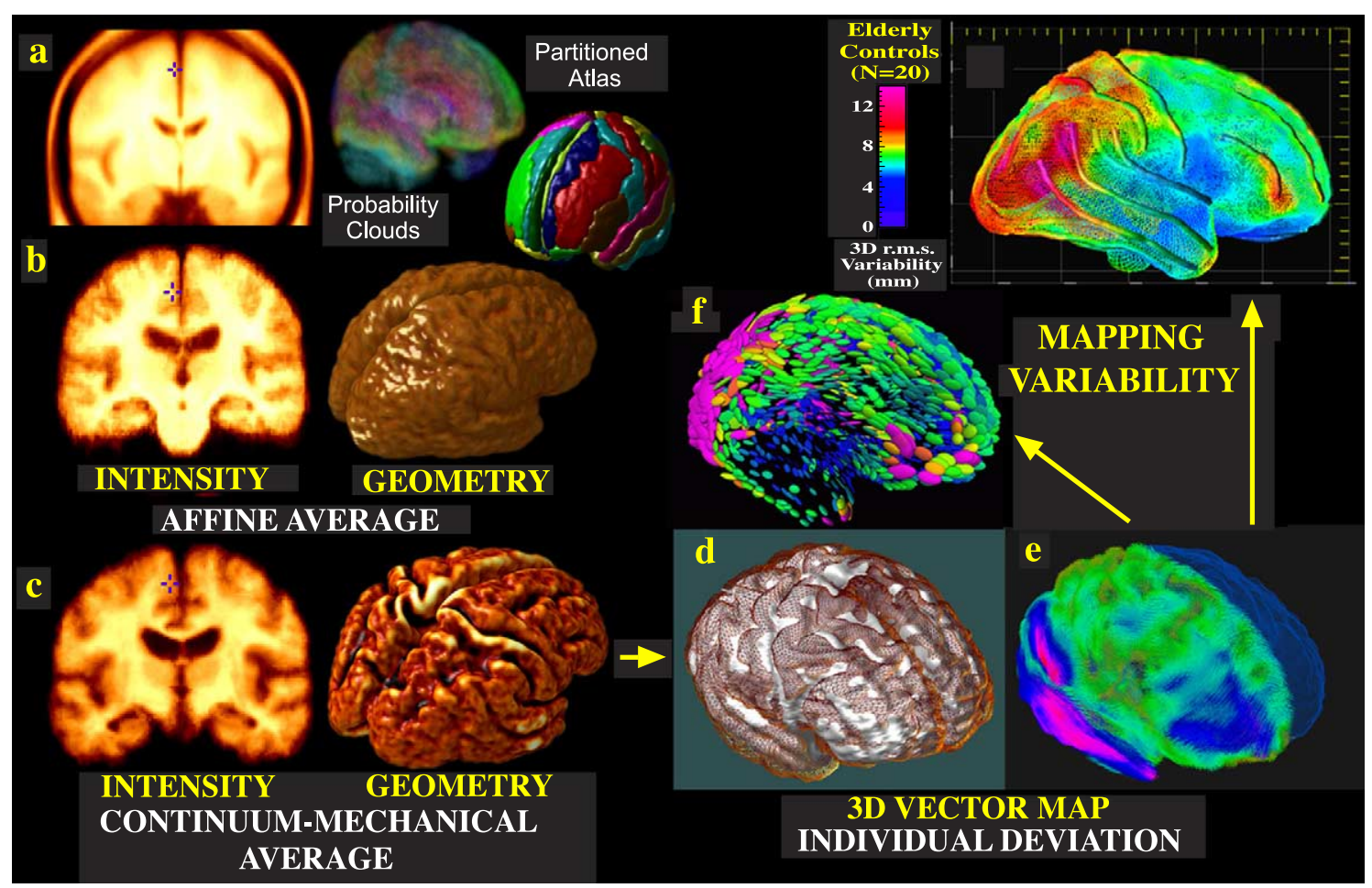

Fig. 1. Averaging brain anatomy. Direct averaging of structural MRI data after a simple affine transform into stereotaxic space washes cortical features away ((a); Evans et al., 1994; $N=305$ normals; (b) shows a similar approach applied to a smaller group of nine Alzheimer's patients). If data are linearly mapped to the $N=$ 305 template in (a), the resulting dispersion of structures in stereotaxic space can be represented by statistical maps ('Probability clouds') that express, at each voxel, the fraction of subjects in which a specific structure occurs. However, a more well-resolved average brain template can be produced [(c); Thompson et al., $2000 \mathrm{a}, \mathrm{b}]$ by averaging a set of surface-based 3D geometric models and warping each subject's 3D scan into the average configuration for the group of subjects. In this approach, 3D deformation vector maps are computed (e) to store individual deviations from a group average (e.g., between the brown surface mesh (d), which represents an individual, and the white surface, which represents the group average anatomy). The local covariance tensor (f) of these 3D vector fields-that deform a set of individual anatomies onto a group average - stores information on the preferred directions and magnitude (g) of anatomic variability that is found in a population (pink colors, large variation; blue colors, less). Ellipsoidal glyphs represent isovalues of probability density for finding anatomy, in a randomly selected individual, that corresponds to a given point on the average cortex (see Thompson et al., 1996a,b, for their derivation). Superquadric glyphs may also be employed to better visualize the local eigenstructure (i.e., preferred directions) of anatomical variation (Kindlmann et al., 2004).

be constructed, and how they can be compared across individuals and groups. We discuss three key steps in creating statistical maps of cortical anatomy: (i) cortical parameterization, or creating geometrical models of the cortical surface; (ii) matching cortical features across individuals, which requires warping one brain surface onto another; and (iii) statistical comparisons to understand effects of disease, aging, or development on anatomy, which can also be used to map group differences or identify correlations between brain structure and genetic or cognitive differences. We show how these methods can be applied to reveal hitherto unknown features of Alzheimer's disease, schizophrenia, and normal development, suggesting their potential in biomedical and clinical research. Finally, we suggest areas where additional mathematical research is likely to speed the pace of discovery in these areas of neuroscience.

\section{Methods}

As discussed above, algorithms to analyze cortical structure and function in diseased populations must inevitably grapple with the anatomic variability that occurs among normal individuals, which makes it difficult to compare data from one subject to another. Fig. 2 shows some processing steps that are carried out in a typical structural neuroimaging study for creating models and maps of the brain. Standard processing steps involve the linear or nonlinear alignment of MRI data from all subjects in a study to a standardized anatomical template, such as an average brain MRI dataset in standardized coordinates (Fig. 2, panel 1). Aligned imaging data are then typically corrected for intensity inhomogeneities and segmented into gray matter, white matter, and CSF (see e.g., Ashburner and Friston, 2000; Fig. 2, panel 2). The simplest and perhaps most intuitive type of analysis then requires the parcellation of the gray and white matter volumes into lobes, or sometimes into finer subdivisions, for regional quantification of tissue volumes (Fig. 2, panels 2a and 2b; see e.g., Giedd et al., 1999; Jernigan et al., 2001; Kennedy et al., 1998, for this type of approach). These brain volume measures can then be compared using standard statistical techniques, such as analysis of variance or multiple regression. More sophisticated analyses allow the creation of maps of anatomical differences. This may involve the extraction of cortical surface models from each image data set as well as the flattening and warping of cortical features on these models to improve the alignment of data from one subject to another (Thompson et al., 1996a,b, 2003). While more global cortical measures may be computed such as surface complexity (Blanton et al., 2001; Luders et al., 2004; Narr et al., 2004a,b; Thompson et al., 1996a,b), it is typically more useful to assess 


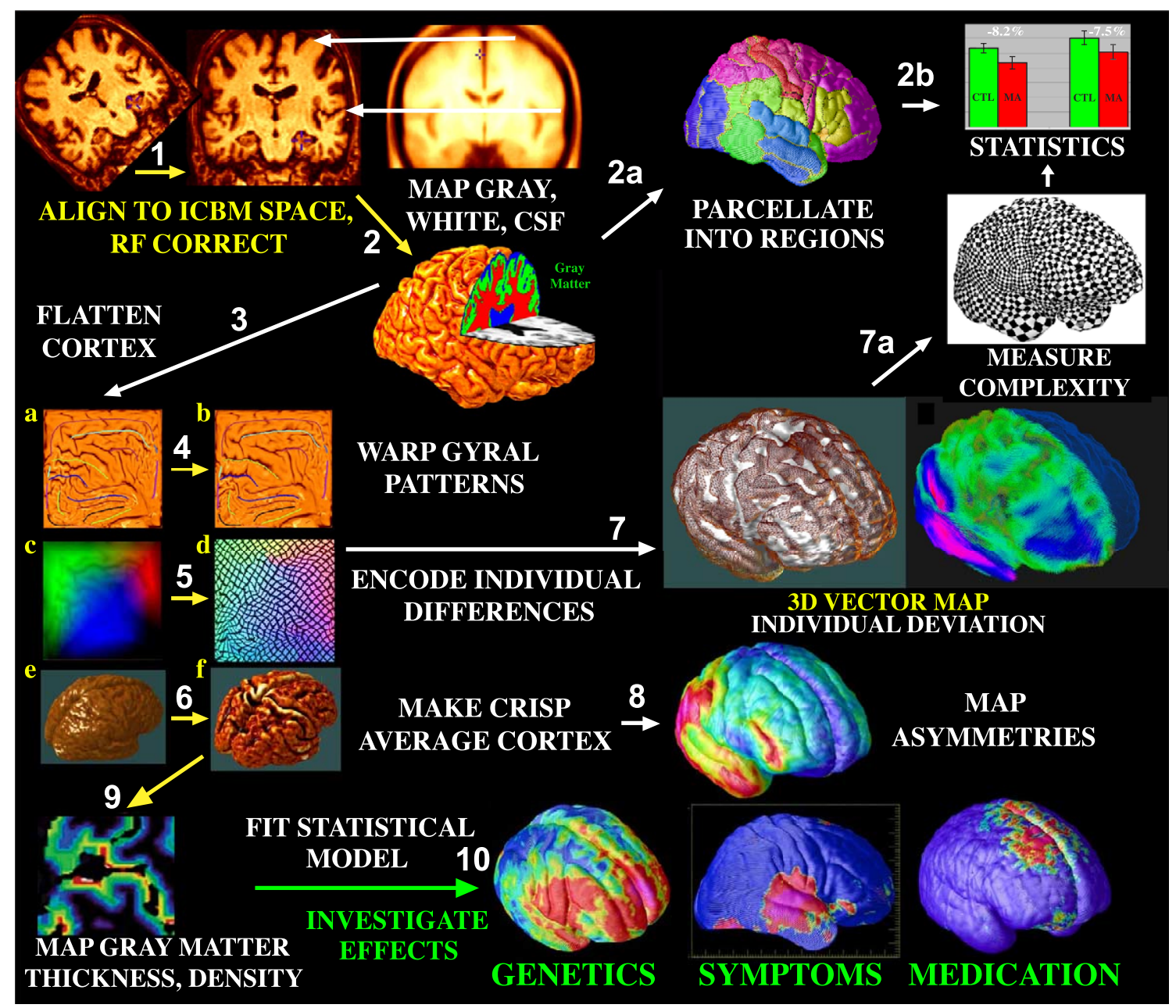

Fig. 2. Image analysis steps for detecting differences in cortical anatomy. An image analysis pipeline is shown here. It can be used to create maps that reveal how brain structure varies in large populations, differs in disease, and is modulated by genetic or therapeutic factors. 3D MRI scans from patients and controls are aligned (1) with an average brain template based on a population (here the ICBM template is used, developed by the International Consortium for Brain Mapping; Mazziotta et al., 2001). Tissue classification algorithms then generate maps of gray matter, white matter, and CSF (2). In a simple analysis, these tissue maps can be parcellated into lobes (2a) and their volumes assessed with analysis of variance or other simple statistics (2b). Or, to compare cortical features from subjects whose anatomy differs, individual cortical surfaces can be flattened (3) and aligned with a group average gyral pattern (4). If a color code indexing 3D cortical locations is flowed along with the same deformation field (5), a crisp group average model of the cortex can be made (6). Relative to this average, individual gyral pattern differences (7), measures of cortical complexity (7b), or cortical pattern asymmetry (8) can be computed. Once individual gyral patterns are aligned to the mean template, differences in gray matter density or thickness (9) can be mapped after pooling data across subjects from homologous regions of cortex. Correlations can be identified between differences in gray matter density or cortical thickness and genetic risk factors (10). Maps may also be generated visualizing regions in which linkages are detected between structural deficits and clinical symptoms, cognitive scores, and medication effects.

cortical differences more locally, computing local measures such as gray matter thickness (Fig. 2, panel 9), gray matter density (GMD; Good et al., 2001; Sowell et al., 1999a,b; Wright et al., 1995), or cortical pattern asymmetry (Sowell et al., 2002a,b,c; Thompson et al., 2001a,b). These are sensitive measures of cortical integrity in a variety of diseases and developmental processes, and changes in these measures are often tightly linked to disease progression and changes in cognition (Sowell et al., 2003a,b; Thompson et al., 2001a,b).

\section{Cortical surface extraction and parameterization}

In high-quality MRI data (typically $1 \times 1 \times 1 \mathrm{~mm}$ image resolution and good tissue contrast is required), it is relatively easy to extract a $3 \mathrm{D}$ cortical surface model (Figs. 3e and f) from an individual subject's scan (Fig. 3d). This represents their cortical surface anatomy in detail (Fig. 3g; triangulated mesh). Two types of methods are common: the first deforms a 3D surface with a fixed parameterization, such as a spherical mesh, into the configuration of the cortex. The deforming surface evolves to match a specified threshold intensity, or isovalue, in the image data while obeying additional constraints that avoid self-intersection and guarantee a smooth deformation of the surface (see, e.g., Davatzikos, 1996; MacDonald, 1998). The second type of approach identifies the white matter surface first as a set of voxels, imposes a surface triangulation on it, and then inflates it to a sphere so that the spherical coordinates can be projected back onto the original surface as a basis for subsequent computations. It can be advantageous if the parametric grid, induced onto the cortex, has desirable mathematical properties. Much work has gone into 


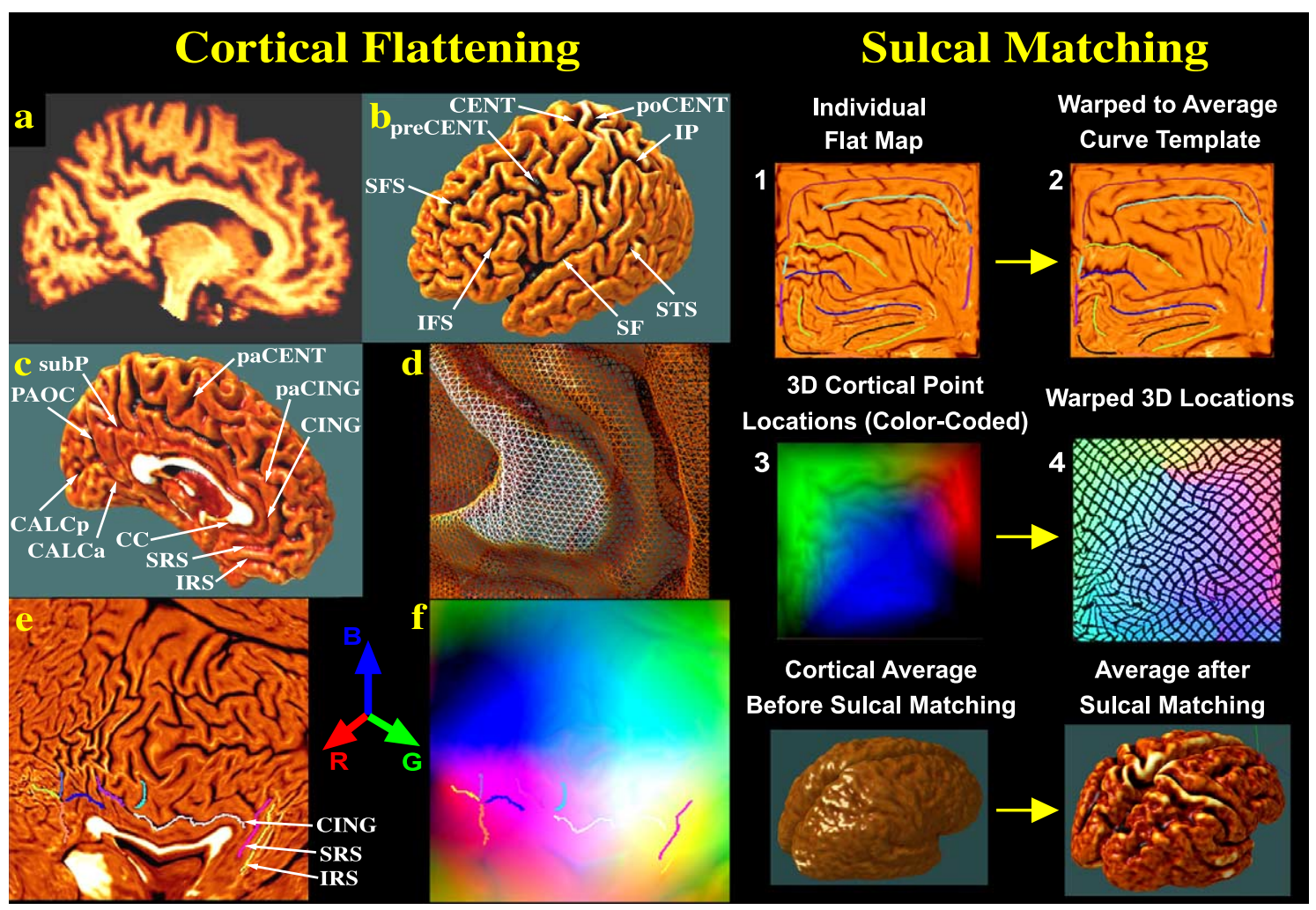

Fig. 3. Cortical pattern matching. Before computing individual anatomical differences, it is useful to create an average model of anatomy for a specific population. If MRI scans from a group of subjects are mutually aligned and their intensities are averaged together pixel-by-pixel (see Fig. 1), cortical features are washed away. To retain these features in the group average, a procedure called cortical pattern matching can be used. From each individual's MRI scan (a) a cortical model (b and c) consisting of discrete triangular elements (d) is created and flattened (panel 1) along with digital models of cortical sulci traced on the brain surface (e). A warping field drives the flat map (1), and a color code indexing corresponding 3D cortical positions ( 3 and 4; also shown in f) to match an average set of flat 2D sulcal curves (2). If these color images are averaged across subjects and decoded before cortical pattern matching (3), a smooth average cortex (5) is produced. If they are warped first (4), averaged, and decoded, a crisp average cortex appears in which anatomical features are reinforced and appear in their mean stereotaxic locations (6). Such cortical averages provide a standard template relative to which individual differences may be measured (Fig. 4). Using warping (4), cortical data can be transferred from individuals whose anatomy is different onto a common anatomic template for comparison and integration.

developing parameterizations that are conformal (Angenent et al., 1999; Haker et al., 2000; Hurdal and Stephenson 2004, this volume); in these, the grid lines on the surface intersect at right angles (see grid in Fig. 2, panel 7a), and angular relationships are preserved as data are mapped from one surface to another. In one approach we developed (Gu et al., 2003; Wang et al., 2004), an arbitrary triangulation of the cortex is mapped onto the sphere, minimizing the harmonic energy of the mapping. This allows spherical coordinates to be projected back onto the original cortices. These coordinates are conformal, and the coordinate frame can be used for subsequent comparisons of structural or functional measures.

\section{Cortical pattern matching and anatomical averaging}

Although cortical parameterization induces a spherical coordinate system onto the cortices of multiple individuals, there is no guarantee that corresponding anatomical landmarks, such as cortical sulci, will occur at the same spherical coordinate across subjects. To achieve this, a higher order anatomical matching procedure is used (cortical pattern matching), which takes models of the sulci in each individual and matches them across subjects. By matching individual cortices to a group average or template, the coordinates of the average or template can be projected back onto each individual subject's surface. If this is performed, data can be compared and averaged across subjects by pooling data that occur at the same surface coordinate location in each subject (see also Fischl et al., 1999, for a related approach, that matches curvature profiles on the sphere).

To perform cortical pattern matching (Thompson and Toga, 1996; Thompson et al., 2000a,b, 2003), we have developed a reliable protocol for manually defining 38 sulcal curves on each subject's cortical surface rendering (Figs. 3e and f), representing each subject's primary gyral pattern. These curves are used as anchors to create a deformation mapping (Fig. 3, panel 2), which distorts the anatomy of one subject onto another, matching sulcal features exactly (to the extent that they are validly defined for each subject). To compute this mapping, cortical models and curves are first flattened (Fig. 3, panel 1), and a flow field is computed in the flattened space to drive individual sulcal features onto an average set of landmark curves (panel 2). Using a mathematical trick, a color code representing 3D locations of cortical points in each subject (panel 3) is convected along with this flow (panel 4). Then these warped color images are averaged across subjects and decoded to produce a crisp average cortical model for the group (panel 6). 
Mathematics of matching: covariant PDEs

To understand how cortical anatomy is matched across subjects, the matching process can be understood in terms of computing a flow field in the cortical parameter space, which matches up corresponding sulci. Cortical models are created by driving a tiled, spherical mesh into the configuration of each subject's cortex, so any point on the cortical surface must map to exactly one point on the sphere and vice versa. These spherical locations, indexed by two parameters, can also be mapped to a planar region $\Omega=[0,2 \pi) \times[0, \pi)$. Cortical differences between any pair of subjects are then expressed as a flow field that elastically warps one flat map onto the flat map of another subject. The parameter shift function $\boldsymbol{u}(\boldsymbol{r}): \Omega \rightarrow \Omega$, is given by the solution $\boldsymbol{F}_{\mathrm{pq}}: \boldsymbol{r} \rightarrow \boldsymbol{r}-\boldsymbol{u}(\boldsymbol{r})$ to a curve-driven warp in the spherical parametric space $\Omega=[0,2 \pi) \times[0, \pi)$ of the cortex. For points $\mathbf{r}=(\boldsymbol{r}, \boldsymbol{s})$ in the parameter space, a system of simultaneous partial differential equations can be written for the flow field $\boldsymbol{u}(\boldsymbol{r})$ :

$$
\begin{aligned}
& L^{\ddagger}(\boldsymbol{u}(\boldsymbol{r}))+\boldsymbol{F}(\boldsymbol{r}-\boldsymbol{u}(\boldsymbol{r}))=\mathbf{0}, \forall \boldsymbol{r} \in \Omega, \text { with } \boldsymbol{u}(\boldsymbol{r}) \\
& \quad=\boldsymbol{u}_{0}(\boldsymbol{r}), \forall \boldsymbol{r} \in M_{0} \cup M_{1}
\end{aligned}
$$

Here $\boldsymbol{M}_{0}, \boldsymbol{M}_{1}$ are sets of points and (sulcal or gyral) curves where displacement vectors $\boldsymbol{u}(\boldsymbol{r})=\boldsymbol{u}_{0}(\boldsymbol{r})$ matching corresponding anatomy across subjects are known. The flow behavior is modeled using equations derived from continuum mechanics, and these equations are governed by the Cauchy-Navier differential operator $\boldsymbol{L}=\boldsymbol{\mu} \nabla^{2}+(\boldsymbol{\lambda}+\boldsymbol{\mu}) \nabla\left(\nabla^{\mathrm{T}} \bullet\right)$ with body force $\boldsymbol{F}$ (Avants and Gee, 2004; Davatzikos et al., 1996; Miller, in press; Thompson et al., 2000a,b; this volume). The only difference is that $\boldsymbol{L}^{\ddagger}$ is the covariant form of the differential operator $\boldsymbol{L}$ (for reasons explained below). This approach not only guarantees precise matching of cortical landmarks across subjects, but also creates mappings that are independent of the surface metrics, and therefore independent of the surface parameterizations.

For those not familiar with continuum mechanics, this process can be thought of as minimizing a mathematically defined measure of distortion as one flat map is distorted onto another. Because the sulci are matched exactly, the remainder of the map must be distorted, although there are infinitely many mappings that are consistent with the sulci being matched. To pick a unique one, the mapping that minimizes a distortion measure is selected. This distortion can be expressed using an operator, here denoted by $\boldsymbol{L}$, which is typically the CauchyNavier differential operator $\boldsymbol{L}=\boldsymbol{\mu} \nabla^{2}+(\boldsymbol{\lambda}+\boldsymbol{\mu}) \nabla\left(\nabla^{\mathrm{T}} \boldsymbol{\bullet}\right)$, or powers of the Laplacian on space or space time (Christensen et al., 1996; Dupuis et al., 1998; Grenander and Miller, 1998; Joshi et al., 1998; Toga, 1998; Toga and Thompson, 2003a,b). These operators tend to penalize high values of the Laplacian $\nabla^{2} \boldsymbol{u}(\boldsymbol{r})$ of the flow, as well as high values of the gradient of the divergence of the flow $\nabla\left(\nabla^{\mathrm{T}} \boldsymbol{u}(\boldsymbol{r})\right)$. If the Laplacian term is used, it prohibits sharp spatial changes in the gradient of the mapping (i.e., large second derivatives) along straight lines; if the latter term is used, it keeps the areal dilatation of the mapping as uniform as possible.

Covariant mapping equations. Because the cortex is not a developable surface, it cannot be given a parameterization whose metric tensor is uniform. As in fluid dynamics or general relativity applications, the intrinsic curvature of the solution domain should be taken into account when computing flow vector fields in the cortical parameter space and mapping one mesh surface onto another. If not, the specific triangulations of the surfaces will affect how the surfaces are matched. In the covariant PDE approach (Christensen et al., 1996; Dupuis et al., 1998; Grenander and Miller, 1998; Joshi et al., 1998; Thompson et al., 2000a,b; Toga, 1998; Toga and Thompson, 2003a,b), correction terms (Christoffel symbols, $\Gamma_{j k}^{i}$ ) make the necessary adjustments for fluctuations in the metric tensor of the mapping procedure. In the partial differential equations (Eq. (1)), we replace $L$ by the covariant differential operator $L^{\ddagger}$. In $L^{\ddagger}$, all $L$ 's partial derivatives are replaced with covariant derivatives. These covariant derivatives are defined with respect to the metric tensor of the surface domain where calculations are performed. The covariant derivative of a (contravariant) vector field $u^{i}(x)$ is defined as $u^{i}{ }_{, k}=\partial u^{j} / \partial x^{k}+\Gamma^{j}{ }_{i k}$ $u^{i}$ where the Christoffel symbols of the second kind (Einstein, 1914) $\Gamma_{i k}^{j}$ are computed from spatial derivatives of the metric tensor components $g_{j k}(x)$ :

$\Gamma_{j k}^{i}=(1 / 2) g^{i l}\left(\partial g_{l j} / \partial x^{k}+\partial g_{l k} / \partial x^{j}-\partial g_{j k} / \partial x^{i}\right)$.

These correction terms are then used in the solution of the Dirichlet problem (Joshi et al., 1995) to match one cortex with another. Note that a parameterization-invariant variational (integral) formulation could also be used to minimize metric distortion of data when mapping them from one surface to another. If $P$ and $Q$ are cortical surfaces with metric tensors $g_{j k}\left(u^{i}\right)$ and $h_{j k}\left(\xi^{\alpha}\right)$ in local coordinates $u^{i}$ and $\xi^{\alpha}(i, \alpha=1,2)$, the Dirichlet energy of the mapping $\xi(u)$ is defined as: $E(\xi)=\int_{P} e(\xi)(u) \mathrm{d} P$, where $e(\xi)(u)=g^{i j}(u) \partial \xi^{\alpha}(u) /$ $\partial u^{i} \partial \xi^{\beta}(u) / \partial u^{j} h_{\alpha \beta}(\xi(u))$ and $\mathrm{d} P=\left(\sqrt{ } \operatorname{det}\left[g_{i j}\right]\right) \mathrm{d} u^{1} \mathrm{~d} u^{2}$. The Euler equations, whose solution $\xi^{\alpha}(u)$ minimizes the mapping energy, are (Liseikin, 1991)

$$
\begin{aligned}
0 & =L\left(\xi^{i}\right) \\
& \left.=\sum_{m=1 \text { to } 2} \partial / \partial u^{m}\left[\left(\sqrt{\operatorname{det}\left[g^{r u}\right]}\right)\right] \sum_{l=1 \text { to } 2} g_{u r}^{m l} \partial \xi^{i} / \partial u^{l}\right](i=1,2),
\end{aligned}
$$

The resulting (harmonic) map (1) minimizes the distortion as data are mapped from one surface to the other, and (2) is again independent of the parameterizations (spherical or planar) used for each surface. Related algorithms for minimizing harmonic energies, invariant under reparameterization, have been developed in modeling liquid crystals (Alouges and Ghidaglia, 1997) and in Polyakov's formulation of string theory (Polyakov, 1987). Harmonic mappings or their more general counterparts $p$-harmonic maps (Joshi et al., 2004) are beginning to see widespread application in brain mapping due to their ability to map complex surfaces onto simpler objects such as 3D spheres or 2D planes while minimizing spatial distortions (Joshi et al., 2004; Wang et al., 2004).

Operator learning. Because of the range of operators available to regularize deformation mappings (Cachier and Ayache, 2004; Joshi et al., 2004), the question arises as to which is the most appropriate to use in brain mapping (this may differ for different applications, such as aligning functional landmarks across subjects, or mapping brain growth over time). If large databases of landmarks are available, the regularization operator $L$ can actually be learned using spectral estimation (Grenander and Miller, 1998). Alternatively, the Green's function (impulse response function) of the 
operator can be estimated from the spatial autocorrelation of the mappings of landmarks. Cachier and Ayache (2004) developed a technique to find all isotropic differential quadratic forms of any order on vector fields. These give rise to an extremely general class of vector regularization and filtering techniques that can be applied in the Fourier domain. Even so, the optimal regularization operators in brain mapping are not likely to be spatially stationary (i.e., they ought to have different values in different brain regions); this is because the covariance tensor of brain deformation fields is highly heterogeneous and anisotropic (see Fig. 1; Thompson et al., 2001a,b).

Implicit functions. The above scheme for surface matching is complicated to implement due to the need to maintain information on surface triangulations and compute numerical derivatives of quantities such as the surface Laplacians of fields defined on surfaces, and the components and Christoffel symbols of the surface metric. Intriguingly, the approach can be greatly simplified if distance functions to the surfaces are computed in $3 \mathrm{D}$ (the socalled 'level set' approach; Osher and Sethian, 1988). After some mathematical manipulation of the PDEs (Memoli et al., 2002, in press, this volume), all computations can be performed in the $3 \mathrm{D}$ image, which eases numerical implementations. Specifically, a mapping is found that minimizes the harmonic map functional defined by $E[u]=1 / 2 \int_{\mathrm{s}}\left\|J_{u}\right\|^{2} \mathrm{~d} S$, when the norm is the norm of Frobenius and $J_{u}$ is the Jacobian of the mapping taking the source surface $S$ onto the level-set (zero isovalue) of the target surface, $u(x, y): S \rightarrow\{\phi=0\}$. Memoli et al. (2002, in press) showed that the corresponding gradient descent is given by ( $H$ stands for the Hessian) $\frac{\partial u}{\partial t}=\Delta u+\left(\sum_{k} H_{\phi}\left[\frac{\partial u}{\partial x_{k}}, \frac{\partial u}{\partial x_{k}}\right]\right)$, with the advantage that all derivatives are computed on $3 \mathrm{D}$ distance fields, rather than on complicated surface triangulations (Fig. 4).

\section{Statistical maps on the cortex}

Cortical pattern matching is simply a nonlinear registration method for pooling data across subjects. These data may include information on cortical thickness, gray matter density, functional MRI signals (Rasser et al., in press; Zeineh et al., 2003), or many other cortical measures. Fig. 5 shows a variety of types of cortical measures that can be plotted onto the cortex. In general, these measures are aligned across subjects using the cortical pattern matching procedure. A statistical model, such as the general linear model, is then fitted at each cortical surface point (as in packages for functional image analysis, such as the Statistical Parametric Mapping software; Friston et al., 1995). Each cortical measure is sensitive to a different attribute of cortical anatomy, and each can be used to monitor developmental or disease processes or identify group differences in brain structure.

\section{Cortical thickness}

In Fig. 5, panels a-d show steps involved in measuring cortical thickness (for related work, see Annese et al., 2002; Fischl and Dale, 2000; Jones et al., 2000; Kruggel et al., 2001; Miller et al., 2000; Yezzi and Prince, 2001). In our approach, the MRI scan (a) is classified into gray matter, white matter, CSF, and a background class (respectively represented by green, red, black, and white colors in b). To quantify cortical gray matter thickness, we use the 3D distance measured from the cortical white-gray matter boundary in the tissue-classified brain volumes to the cortical surface (gray-CSF boundary) in each subject (c). Tissue-classified brain volumes are first resampled to $0.33-\mathrm{mm}$ isotropic voxels to obtain distance measures indexing gray matter thickness at subvoxel spatial resolution. Gray matter thickness, measured at thousands of homologous cortical locations in each subject, is then compared across subjects and averaged at each cortical surface location providing spatially detailed maps of local thickness differences within or between groups. The thickness data may also be smoothed using a surface-based kernel to enhance signal to noise before making cross-subject comparisons. Panel d shows the mean cortical thickness in a group of 40 healthy young adults, ranging from low values in primary sensorimotor and visual cortices $(2-3 \mathrm{~mm}$, yellow colors) to highest values on the medial wall in cingulate areas (up to $6 \mathrm{~mm}$, purple colors). The regional variations in these maps agree with those found in the classical cortical thickness maps derived postmortem by von Economo (see Sowell et al., in press,b).

\section{Gray matter density}

Because thickness maps are relatively difficult to derive, most work mapping gray matter differences in the last 10 years has focused on mapping gray matter density (GMD; Ashburner and Friston, 2000; Sowell et al., 1999a,b; Thompson et al., 2003; Wright et al., 1995). GMD is quite highly correlated with cortical thickness (see Narr et al., 2004a,b, for a map we created correlating the two measures) and it can also be used to quantify gray matter in subcortical structures. Mathematically, a measure $g_{i, r}(x)$ can be defined as the 'gray matter density', that is, the proportion of voxels classified as gray matter falling within a sphere (center $x$, radius $r$ ) in the $i$ th subject's scan. Due to the cortical pattern matching, we also have a family of $3 \mathrm{D}$ deformation maps $U_{i}(r)$ matching each individual cortex in $3 \mathrm{D}$ to the average cortex for a group. Here $U_{i}$ is a $3 \mathrm{D}$ location on the $i$ th subject's cortex and $r$ is the location it maps to, after warping, in the cortical parameter space. Then for a point at parameter location $r$ on the group average cortex (Fig. 3, panel 6), $g_{i, r}\left(U_{i}(r)\right)$ is the gray matter density at the corresponding cortical point in subject $i$. A statistical model is fitted, at each surface vertex, to assess group differences in GMD and the mean percent difference and its significance can be shown as color-coded maps. For example, Figs. 5e and $\mathrm{f}$ show the mean GMD in a group of 21 normal subjects and 22 chronic methamphetamine users, respectively (data are from Thompson et al., 2004a). The 3-5\% mean reduction in the methamphetamine group is shown in $g$, where red colors denote greater GMD deficits. In h, the significance of these reductions is mapped, with red colors showing regions significant at the $P<0.05$ level. To correct the result for multiple comparisons (implicit in carrying out statistical tests at a large number of surface vertices), a permutation test is used (Edgington, 1969, 1995; Nichols and Holmes, 2002; Thompson et al., 2003; other approaches based on false discovery rate or random field theory could also be used; Worsley et al., 1999). In the permutation test, a null distribution is built for the area of the cortex exceeding a fixed primary threshold (usually 0.05 ) in the maps, when subjects are randomly assigned to groups. This allows a corrected $P$ value to be derived for the whole map (here it is also 0.05 , but it usually differs from the primary threshold). This quantifies the level of surprise in seeing the pattern of deficits, relative to the null hypothesis of no group differences.

\section{Gyral pattern variability}

Anatomical variability can also be studied using deformation mappings that transform individual anatomies onto a group average 

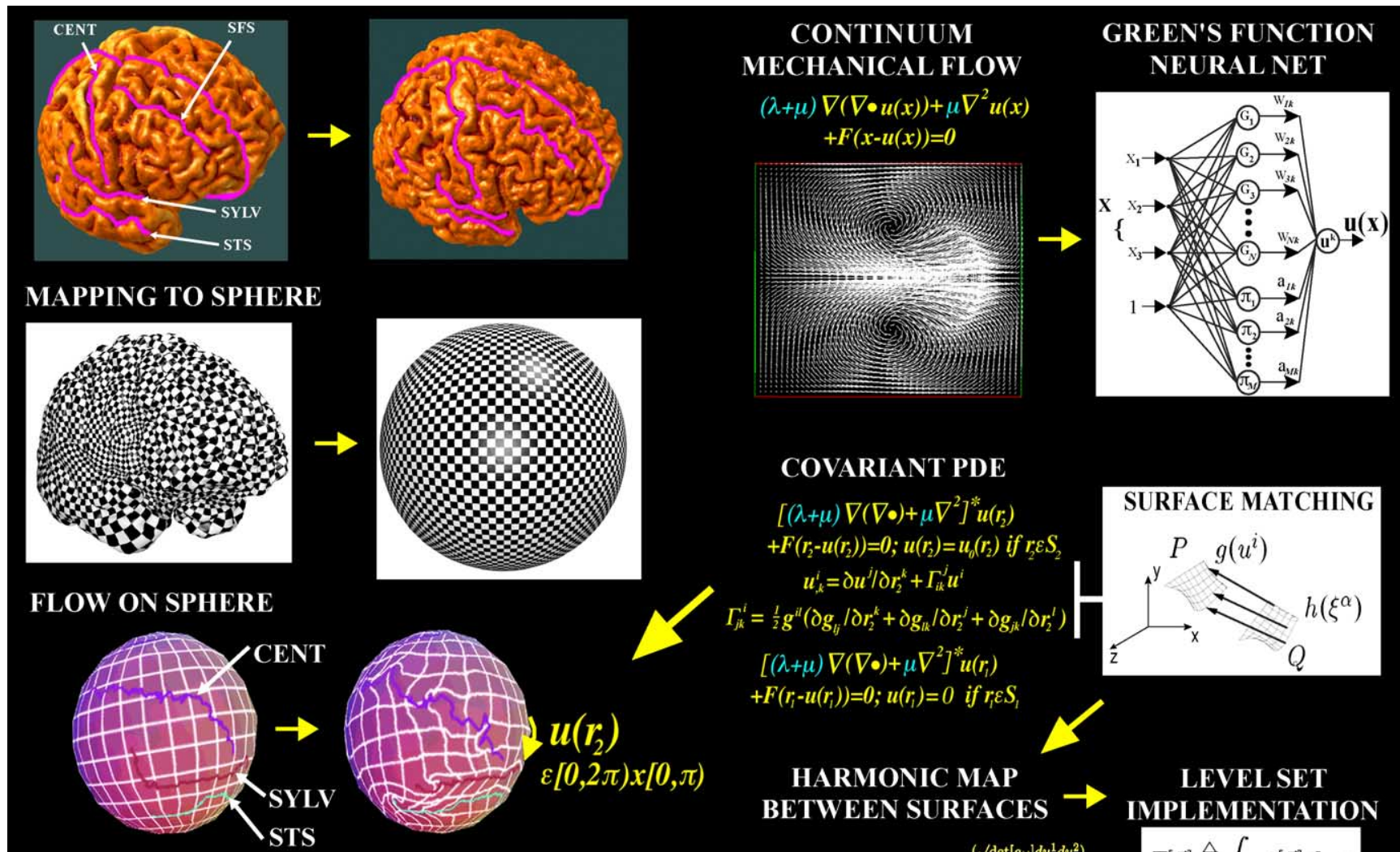

COVARIANT PDE

$\left[(\lambda+\mu) \nabla(\nabla \bullet)+\mu \nabla^{2}\right]^{*} u\left(r_{2}\right)$

$+F\left(r_{2}-u\left(r_{2}\right)\right)=0 ; u\left(r_{2}\right)=u_{0}\left(r_{2}\right)$ if $r_{2} \varepsilon S_{2}$

$u_{i, k}^{i}=\partial u^{j} / \partial r_{2}^{k}+\Gamma_{i k}^{j} u^{i}$

ORIGAMI BOX DISCRETIZATION
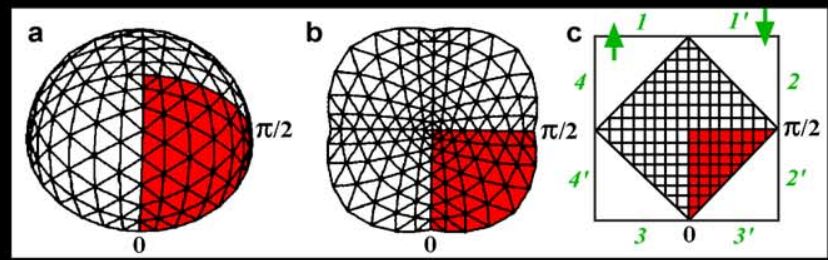

$+F\left(r_{i}-u\left(r_{i}\right)\right)=0 ; u\left(r_{i}\right)=0$ if $r_{i} \varepsilon S$

\section{HARMONIC MAP BETWEEN SURFACES}

$E(\xi)=\int_{P} \frac{e(\xi)(u)}{\uparrow} d P^{\left(\frac{V}{\top} \operatorname{det}\left[g_{i j}\right] d u^{1} d u^{2}\right)}$ $g^{i j}(u) \partial \xi^{\alpha}(u) / \partial u^{i} \partial \xi^{\beta}(u) / \partial u^{j} h_{\alpha \beta}(\xi(u))$ $0=L\left(\xi^{i}\right)=\sum_{m=1}^{2} \partial / \partial u^{m}\left[\operatorname{det}\left(g^{r u}\right) \sum_{l=1}^{2} g_{u r}^{m l} \partial \xi^{i} / \partial u^{l}\right]$ $\rightarrow \xi^{\alpha}(u)$ LEAST DISTORTION INVARIANT
SURFACE MATCHING
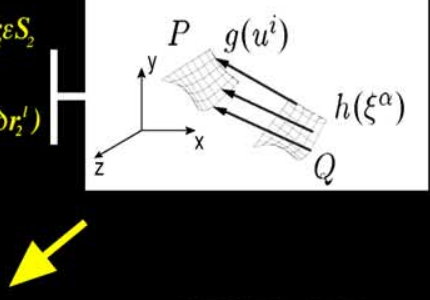

LEVEL SET IMPLEMENTATION

Fig. 4. Connections among mathematical concepts used in surface matching. This schematic illustrates some connections among concepts used in the nonlinear registration (matching) of brain surfaces. Related concepts have been developed in the theory of artificial neural networks and string theory (Thompson et al., $2000 \mathrm{a}, \mathrm{b})$. When matching two anatomical surfaces, it is often desirable not only to match the entire 3D surface of one subject with another, but also to enforce some higher-order anatomical constraints, such as the exact matching of a range of corresponding sulcal curves (or other landmark points, curves, or regions, such as functional landmarks, if these are known). This matching process is simplified by mapping the surface to a sphere (Mapping to Sphere; Wang et al., 2003) and then computing a flow field $u(r)$ that matches features on the sphere (Flow on Sphere; Thompson and Toga, 1996). This flow can be represented by spherical harmonics (Thompson and Toga, 1996; Leow et al., 2004), which are eigenfunctions of the spherical Laplacian, or by solving an elastic or fluid PDE that aligns sulcal/gyral landmarks (Covariant PDE; Bakircioglu et al., 1999; Thompson et al., 2000a,b) or curvature maps (Fischl et al., 1999). The flow equations can be discretized on a spherical or planar domain. These domains can be regarded topologically equivalent if the boundaries of the planar domain are regarded as folded up and logically identified when computations are performed (here called an 'Origami Box' Discretization, by analogy with paper folding). To facilitate the discretization of PDEs on the surface, spherical coordinates (a) are replaced by a flat square 2D multigrid structure (of side $\pi$; $c$ ). In this data structure, no cuts are introduced: connectivity information is retained between boundary nodes that are adjacent on the 3D brain surface (e.g., 1 matches $1^{\prime}$, 2 matches $2^{\prime}$, etc.). Green arrows denote points that are topographically adjacent. In this scheme, cortical points with spherical coordinates $(\theta, \phi)$ lying in the octant $[0, \pi / 2] \times[0, \pi / 2]$ (colored red, a) map to the 2D parameter space location $(\theta, \phi(\pi-2 \theta) / \pi$ ) (red triangle, c). Other mappings are then determined by symmetry. These warping methods originate in equations used in continuum mechanics to describe fluid flows or elastic deformations (Continuum Mechanical Flow; the inset box shows a 2D vector field, or elastostatic flow, computed from the Cauchy-Navier equations, also shown). These flows can actually be computed using neural networks (Davis et al., 1996) exploiting the duality between landmark-driven flows and radial basis function neural nets. In these networks, the hidden units of the neural network are replaced by the Green's functions of the continuum-mechanical operator (Green's Function Neural Net). The matching of surfaces can also be viewed as the distortion of one surface onto another that minimizes an energy defined by an integral (Surface Matching). If the data are triangulated, the harmonic energy can be differentiated to produce an Euler-Lagrange equation that can be iterated to find the map (Harmonic Map between Surfaces). Better still, the surfaces can be defined in terms of distance fields (or other implicit functions) and the matching energy can be discretized using functionals that take zero values off the zero level set of the two manifolds (Level Set Implementation; Memoli et al., 2002, 2004, this volume). Despite differences in formulation, these methods are all fundamentally similar in that they all aim to solve self-adjoint second-order PDEs using either implicit functions, eigenfunction methods, or radial basis function neural nets (see also Toga, 1998 for a book reviewing nonlinear registration approaches used in brain imaging). 


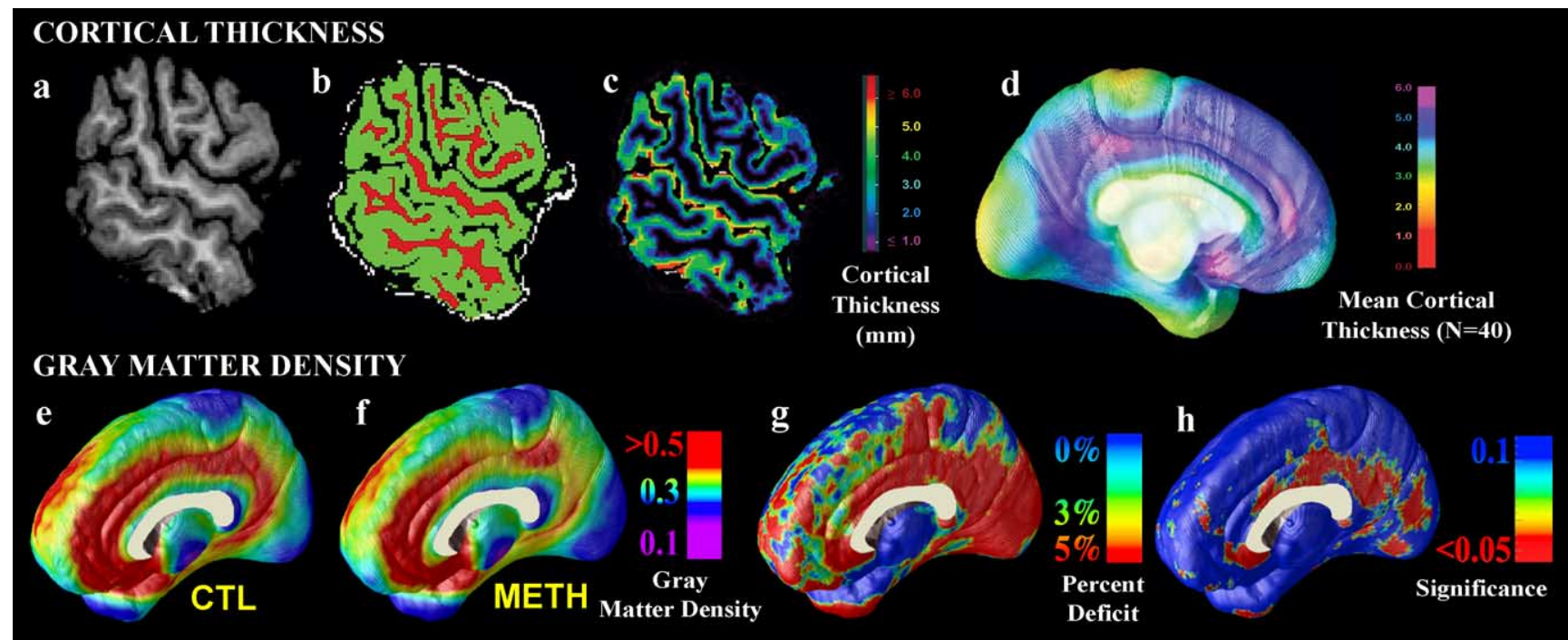

GYRAL PATTERN VARIABILITY
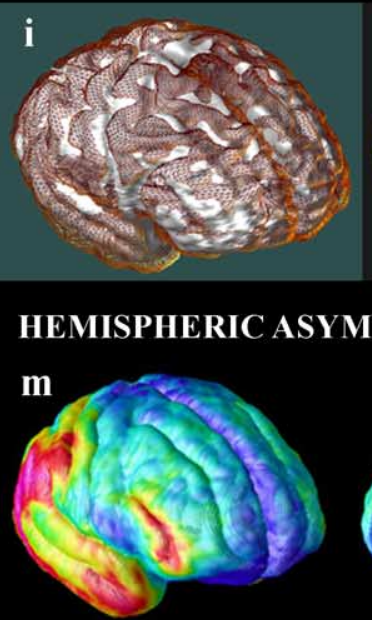

\section{HERITABILITY}

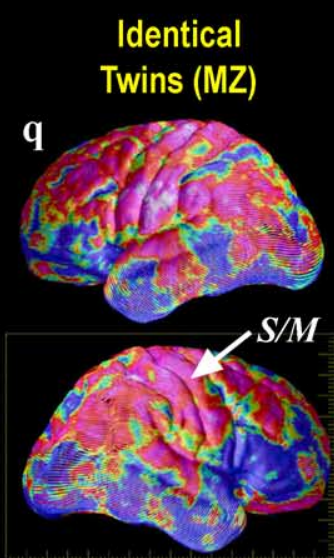

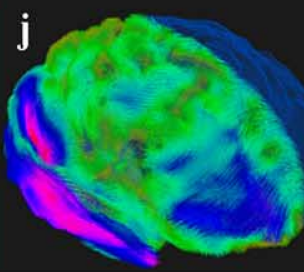
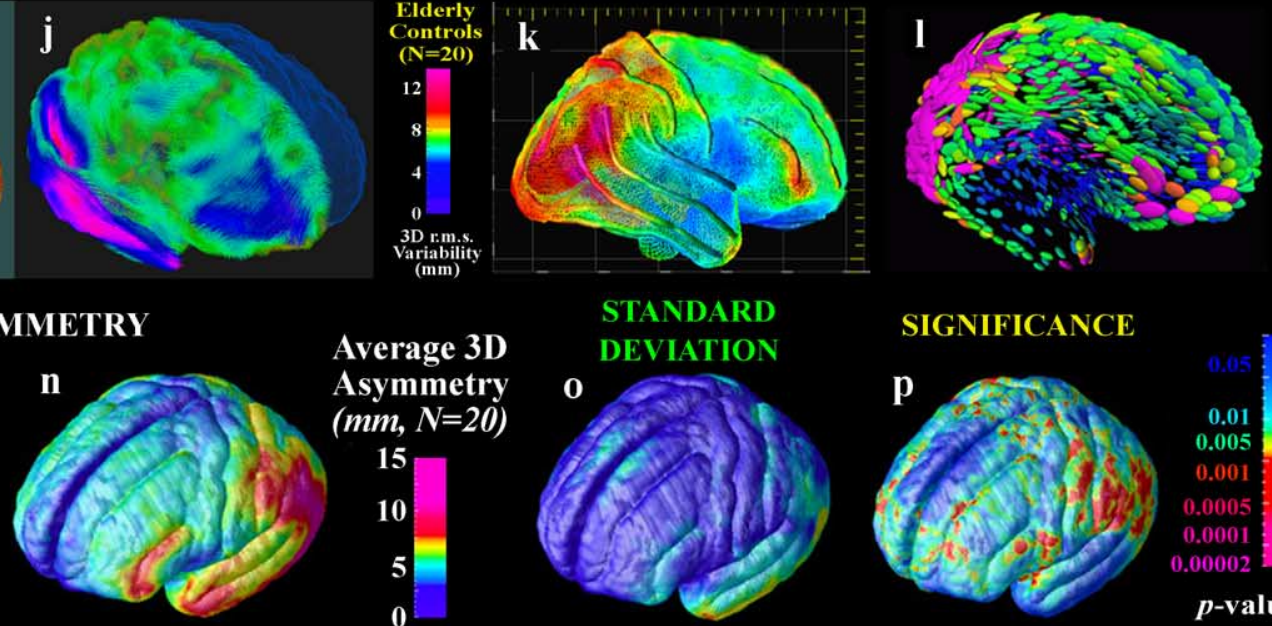

STANDARD

DEVIATION
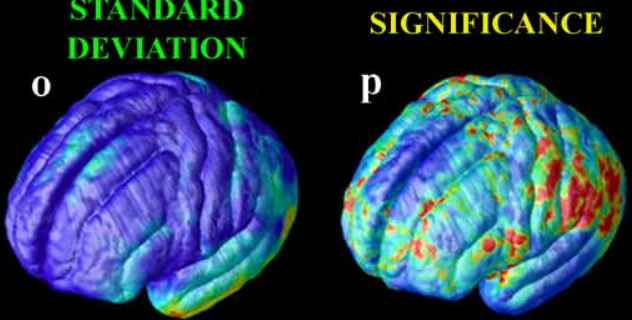

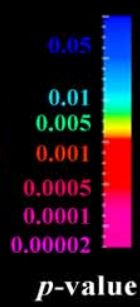

Share all their genes Share half their genes

Identical

Twins (MZ)

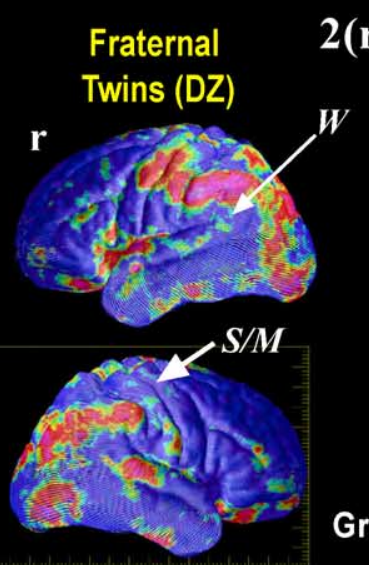

G

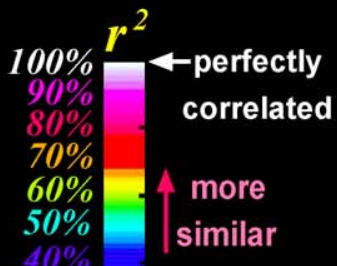

between Twins

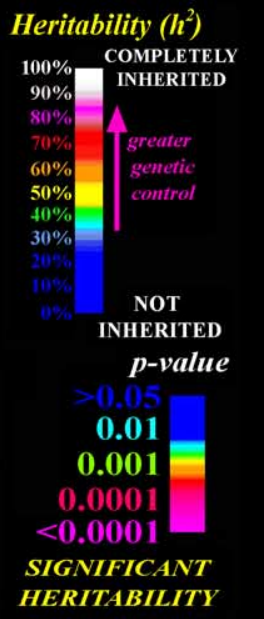

Fig. 5. Statistical maps of cortical structure. A variety of maps can be made that describe different aspects of cortical anatomy. These include maps of cortical thickness $(\mathrm{a}-\mathrm{d})$, gray matter density $(\mathrm{e}-\mathrm{h})$, gyral pattern variability $(\mathrm{i}-1)$, hemispheric asymmetry (m-p), and heritability of brain structure (q-t). Explanations of these features are in the main text. These maps are sensitive to changes in development or disease and can be used to pinpoint regions where structure is abnormal or where it correlates with clinical or treatment parameters. 
(Thompson and Toga, 2002). For example, the cortical pattern matching procedure also computes $3 \mathrm{D}$ deformation mappings that elastically transform each subject's gyral pattern and their entire surrounding cortical surface, onto a group average gyral pattern so that the sulci manually defined in each subject match exactly. These are the cortical pattern differences that remain after the brains are rotated, translated, and scaled to match an average brain template using a linear transformation to match the overall size of the brains. An example of the subsequent 3D deformation mapping, which also matches the brain surfaces and internal sulcal landmarks, is shown in Fig. $5 \mathrm{j}$. This is a $3 \mathrm{D}$ vector field that deforms an individual cortex (brown mesh, i) onto a group average cortex (white surface, i). In j, the magnitude of the deformation vectors is shown in color (pink colors denote large deformations). By taking the root mean square (rms) magnitude of the $3 \mathrm{D}$ deformation vectors from the average surface to the individual subjects, a measure of the $3 \mathrm{D}$ variability of the gyral pattern can be plotted in color on the cortex (Fig. 5k). Perisylvian language areas are the most variable (red colors; up to 12 $\mathrm{mm}$ rms variation) while primary sensorimotor regions hardly vary at all (blue colors; 0-5 mm rms variability; Thompson et al., $2000 a, b)$. After the affine components of the deformation fields are factored out, the deformation vector required to match the structure at position $x$ in the average cortex with its counterpart in subject $i$ can be modeled as:

$W_{i}(x)=\mu(x)+\Sigma(x)^{1 / 2} \varepsilon_{i}(x)$.

Here $\mu(x)$ is the mean deformation vector for the population (which approaches the zero vector for large $N$ ), $\Sigma(x)$ is a nonstationary, anisotropic covariance tensor field estimated from the mappings, $\Sigma(x)^{1 / 2}$ is the upper triangular Cholesky factor tensor field, and $\varepsilon_{i}(x)$ can be modeled as a trivariate random vector field whose components are independent zero-mean, unit variance, stationary random fields. This $3 \mathrm{D}$ probability distribution makes it possible to visualize the principal directions (eigenvectors) as well as the magnitude of gyral pattern variability, and these characteristics are highly heterogeneous across the cortex. For any desired confidence threshold $\alpha, 100(1-\alpha) \%$ confidence regions for possible locations of points corresponding to $x$ on the average cortex are given by nested ellipsoids $E_{\lambda(\alpha)}(x)$ in displacement space (Fig. 51; pink colors denote regions of high variability, blue colors low variability; the color code indicates the determinant of the covariance tensor). Here $E_{\lambda}(x)=\left\{\mu(x)+\lambda[\Sigma(x)]^{-1 / 2} p \mid \forall p \in\right.$ $S(0 ; 1)\}$, where $S(0 ; 1)$ is the unit sphere in $R^{3}$, and $\lambda(\alpha)=[[N(N-3) /$ $\left.\left.3\left(N^{2}-1\right)\right]^{-1} F_{\alpha, 3, N-3}\right]^{1 / 2}$, where $F_{\alpha, 3, N-3}$ is the critical value of the $F$ distribution such that $\operatorname{Pr}\left\{F_{3, N-3}>F_{\alpha, 3, N}-3\right\}=\alpha$, and $N$ is the number of subjects (Thompson et al., 1996a,b, 1997). This type of probabilistic modeling of deformation fields can be used to map patterns of abnormal brain structure (Thompson et al., 1997). In related work, the deformation fields have been modeled as Hotelling's $\mathrm{T}^{2}$-distributed random fields (Cao and Worsley, 1999), or have been subjected to singular value decomposition to identify stereotypical modes of brain deformation (Ashburner et al., 1998; Woods, 2003).

\section{Hemispheric asymmetry}

By analysis of variance in 3D deformation fields that match different subjects' anatomies, it is also possible to map the pattern of brain asymmetry in a group (Thompson et al., 1998; Thirion et al., 2000; Lancaster et al., 2003). Cortical pattern matching is first applied to transform corresponding features in each hemisphere to the same location in parameter space. 3D deformation fields are then recovered matching each brain hemisphere with a reflected version of the opposite hemisphere. The parameter flows are advantageous in that the asymmetry fields are also registered; in other words, asymmetry measures can be averaged across corresponding anatomy at the level of the cortex. This is not necessarily the case if warping fields are averaged at the same coordinate locations in stereotaxic space (cf. Fig. 1a). The pattern of mean brain asymmetry for a group of 20 subjects is shown in Figs. $5 \mathrm{~m}$ and $\mathrm{n}$. The resulting asymmetry fields $a_{i}(r)$ (at parameter space location $r$ in subject $i$ ) were treated as observations from a spatially parameterized random vector field, with mean $\mu_{a}(r)$, and a nonstationary covariance tensor $\Sigma_{\mathrm{a}}(r)$ (cf. Fig. 51). The asymmetry fields are variable themselves, and Fig. 50 shows the $3 \mathrm{D}$ rms magnitude of the asymmetry vector in 20 subjects. Here the temporal lobe exhibits greatest variance in asymmetry, both in regions that have a significant asymmetry and in those that do not. The significance $\alpha$ of deviations from symmetry can be assessed using a $T^{2}$ or $F$ statistic that indicates evidence of significant asymmetry in cortical patterns between hemispheres:

$$
\begin{aligned}
\alpha(r) & =F_{3, N-3}^{-1}\left([(N-3) / 3(N-1)] T^{2}(r)\right) \text { where } T^{2}(r) \\
& =N\left[\mu_{a}(r)^{T} \Sigma_{a}^{-1}(r) \mu_{a}(r)\right] .
\end{aligned}
$$

The brain regions in which significant gyral pattern asymmetries are detected include the perisylvian cortices (red colors; Fig. 5p). In these language-related brain regions, marked hemispheric asymmetries in cortical anatomy are well documented (see Toga and Thompson, 2003a,b, for a review of the literature on structural brain asymmetry).

\section{Heritability}

Another type of cortical map, a genetic brain map (Thompson et al., 2001a,b, 2003) quantifies genetic influences on brain structure. In Thompson et al. (2001a,b), we computed the intraclass correlation in gray matter density $g_{i, r}(x)$ for groups of identical and fraternal twins after cortical pattern matching (giving maps $r_{M Z}(\phi, \theta)$ and $r_{D Z}(\phi, \theta)$ in Figs. 5q and r). In behavioral genetics, a feature is heritable if $r_{M Z}$ significantly exceeds $r_{D Z}$. An estimate of its heritability $h^{2}$ can be defined as $2\left(r_{M Z}-r_{D Z}\right)$, with standard error: $\operatorname{SE}^{2}\left(h^{2}\right)=4\left[\left(\left(1-r_{M Z}^{2}\right)^{2} / n_{M Z}\right)+\left(\left(1-r_{D Z}^{2}\right)^{2} / n_{D Z}\right)\right]$. Fig. $5 \mathrm{r}$ shows a heritability map computed from the equation:

$h^{2}(\phi, \theta)=2\left(r_{M Z}(\phi, \theta)-r_{D Z}(\phi, \theta)\right)$.

Regions in which significant genetic influences on brain structure are detected are shown in the significance map (Fig. $5 \mathrm{t}), p\left[h^{2}(\phi, \theta)\right]$. Genetic influences on brain structure are pronounced in some frontal and temporal lobe regions, including the dorsolateral prefrontal cortex and temporal poles (denoted by DLPFC and T in Fig. 5s). These effects were confirmed by assessing the significance of the effect size of $h^{2}$ by permutation (this involved repeated generation of null realizations of an $h^{2}$-distributed random field; see details in Thompson et al., 2003). Heritable aspects of brain structure are important to identify as they provide endophenotypes to guide the search for specific genes whose variation is linked with deficit patterns in disease or with normal anatomic variation. They are also of interest from a behavioral neuroscience standpoint. The quantity of frontal gray matter, for example, 
is under strong genetic control and is also linked with IQ (Posthuma et al., 2002; Thompson et al., 2001a,b). These genetic brain maps therefore help establish links, at the systems level, between genes, brain structure, and behavior (see Gray and Thompson, 2004, for a review). They also provide a quantitative index of disease liability in those at genetic risk for schizophrenia (Cannon et al., 2002; Narr et al., 2002). With current brain imaging databases, there is now significant power to assess the effects of specific candidate genes on brain development and disease, including alleles that are overtransmitted to individuals with dementia (such as the apolipoprotein E4 allele) and to individuals with schizophrenia.

\section{Results}

Next we review some applications of these cortical maps in neuroscientific projects. These methods have been used to reveal the profile of structural brain deficits in studies of dementia (Thompson et al., 2001a,b, 2003), epilepsy (Lin et al., 2004), depression (Ballmeier et al., 2003), childhood and adult-onset schizophrenia (Cannon et al., 2002; Narr et al., 2004a,b; Thompson et al., 2001a,b), bipolar disorder (Van Erp et al., 2004), attention-deficit/hyperactivity disorder (Sowell et al., 2003a,b), fetal alcohol syndrome (Sowell et al., 2002a,b,c), Tourette syndrome (Sowell et al., 2004a,b), Williams syndrome (Thompson et al., 2004c), and in methamphetamine abusers (Thompson et al., 2004b). Below we describe some examples selected to illustrate the concepts.

\section{Alzheimer's disease}

Figs. 6a and $\mathrm{b}$ show maps from a longitudinal study of Alzheimer's disease, in which a dynamically spreading wave of gray matter loss was mapped as it spread in the brains of patients with AD (Thompson et al., 2003). We analyzed 52 high-resolution MRI scans of $12 \mathrm{AD}$ patients (age $68.4 \pm 1.9$ years) and 14 elderly matched controls (age $71.4 \pm 0.9$ years) scanned longitudinally (two scans; interscan interval $2.1 \pm 0.4$ years). Maps of gray matter deficits allowed visualization of the loss pattern as it spread over time from temporal and limbic cortices into frontal and occipital brain regions, sparing sensorimotor cortices. The shifting deficits correlated extremely strongly with progressively declining cognitive status $(P<0.0006)$. As shown in Figs. 6a and $b$, cortical atrophy occurred in a well-defined sequence as the disease progressed, again mirroring the temporal sequence of beta-amyloid and neurofibrillary tangle accumulation observed at autopsy (Braak et al., 2000). The trajectory of deficits also appeared to match the sequence of metabolic decline typically observed with positron emission tomography (PET).

Frontal regions, spared early in the disease, showed pervasive deficits later ( $>15 \%$ loss). The maps distinguished different phases of $\mathrm{AD}$ and differentiated $\mathrm{AD}$ from normal aging. Local gray matter loss rates $(5.3 \pm 2.3 \%$ per year in $\mathrm{AD}$ versus $0.9 \pm 0.9 \%$ per year in controls) were faster in the left hemisphere $(P<0.029)$ than the right, at least at this stage of $\mathrm{AD}$. Transient barriers to disease progression appeared at limbic/frontal boundaries. A frontal band (0-5\% loss) was sharply delimited from the limbic and temporoparietal regions that showed the severest deficits in $\mathrm{AD}(>15 \%$ loss). This pattern is consistent with the hypothesis that $\mathrm{AD}$ pathology spreads centrifugally from limbic/paralimbic to higher-order asso- ciation cortices (Mesulam, 2000). The path of these gray matter losses, as they emerge over a period of cognitive decline lasting 1.5 years, is observed in a set of video sequences (see URL, http:// www.loni.ucla.edu/ thompson/AD_4D/dynamic.html for several of these time-lapse movies).

\section{Schizophrenia}

We also applied these approaches to map brain change in schizophrenia. One medication-controlled study revealed a dynamic wave of gray matter loss in the brains of early onset schizophrenic patients as they progress through adolescence (Fig. 6; Thompson et al., 2001a,b). Early deficits in parietal brain regions that support language and associative thinking were found to progress anteriorly in the brain into temporal lobes, supplementary motor cortices, and frontal eye fields over a period of 5 years, consistent with the characteristic neuromotor, sensory, and visual search impairments in the disease. In temporal cortices, including primary auditory regions, severe gray matter loss was absent at disease onset but became pervasive later in the disease. These well-defined dynamic patterns were charted in detail in children scanned repeatedly with magnetic resonance imaging (MRI) over multiyear time spans and were replicated in males and females. The spreading wave of gray matter loss can be seen in a set of animation sequences (see URL, http:// www.loni.ucla.edu/ thompson/MOVIES/SZ/sz.html). Ongoing work aims to better understand these deficits by determining whether they are found in genetically at-risk relatives of patients (Cannon et al., 2002), whether they represent an acceleration of normally occurring brain changes (Gogtay et al., 2004), and whether they are differentially modulated by different types of antipsychotic drugs. The cortical pattern matching approach has also been used to correlate gray matter differences with fMRI measures in tasks that recruit the frontal lobes (Rasser et al., in press) and can be applied to functional imaging data with minor modifications (Zeineh et al., 2003).

\section{Development}

The quest for information on healthy brain development and aging can be significantly advanced by collecting brain images over the entire human life span. Several studies have measured or mapped brain changes in children and adolescents scanned repeatedly during childhood and adolescence (Chung et al., 2001a,b; Giedd et al., 1999; Gogtay et al., 2004; Sowell et al., 1999a,b, 2001, 2002a,b, 2004a,b; Thompson et al., 2000a,b, 2001a,b). The dynamics of brain change across the adult human life span are highly nonlinear (Jernigan et al., 1991; Sowell et al., 2003a,b). To help understand these changes, we recently developed a set of statistical mapping approaches to estimate nonlinear (quadratic) effects of aging on brain structure.

In a recent study (Sowell et al., 2003a,b; Fig. 7d), we used MRI and cortical matching algorithms to map gray matter density (GMD) in 176 normal individuals aged 7-87 years. GMD declined nonlinearly with age, fastest between ages 7 and 60, over dorsal frontal and parietal association cortices, on both the lateral and interhemispheric surfaces. Age effects were inverted in the left posterior temporal region, where GMD gain continued up to age 30 , and then rapidly declined. This was the first study to differentiate the trajectory of maturational and aging effects as they vary over the cortex. Visual, auditory, and limbic cortices, 


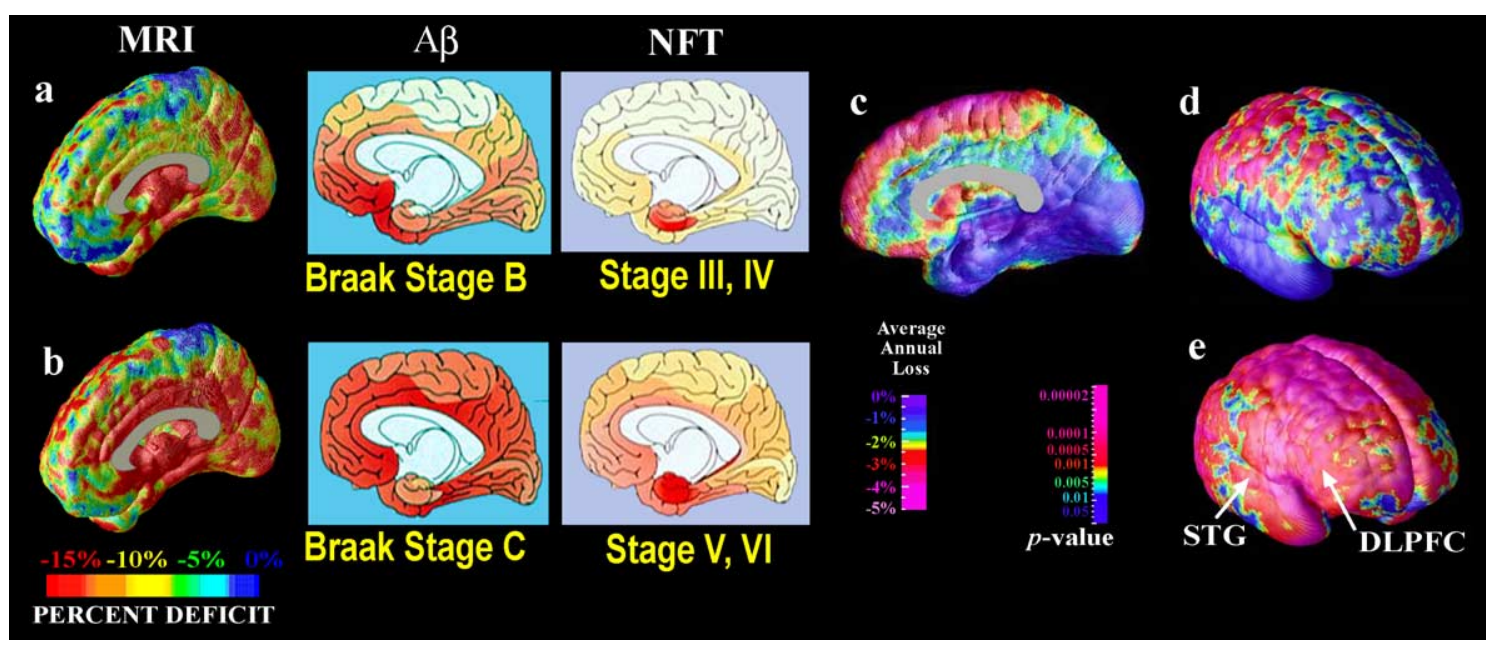

Fig. 6. Dynamic progression of Alzheimer's disease and schizophrenia. Deficits occurring as Alzheimer's disease (AD) progresses are detected by comparing average profiles of gray matter density between $12 \mathrm{AD}$ patients (age: $68.4 \pm 1.9$ years) and 14 elderly matched controls (age: $71.4 \pm 0.9$ years). Patients and controls are subtracted at their first scan (when mean Mini-Mental State Exam (MMSE) score $=18$ for the patients; a) and at their follow-up scan 1.5 years later (mean MMSE $=13$; b). The average percent gray matter loss in patients is shown in a and b. Profound loss engulfs the left medial wall (>15\%) at follow-up. These maps and associated lateral views (Thompson et al., 2003) show a dynamically spreading wave of gray matter loss sweeping forward in the brain from limbic to frontal cortices in concert with cognitive decline. Sensorimotor cortices are relatively spared at both disease stages (blue colors in b). Note the agreement of these MRI-based changes, observed in living patients, with the progression of beta-amyloid (A $\beta$ ) and neurofibrillary tangle (NFT) pathology observed post mortem (Braak Stages B, C and III-VI; adapted from Braak et al., 2000). (c) In a similar longitudinal study of patients with childhood-onset schizophrenia (COS) scanned twice 5 years apart, the frontal cortex underwent a selective rapid loss of gray matter (up to $3-4 \%$ per year faster in patients than controls). Subtraction maps contrasting patients with controls revealed early deficits in parietal regions (red colors; d) that spread forward into the rest of the cortex at follow-up (e; superior temporal gyrus (STG) and the dorsolateral prefrontal cortex (DLPFC) are indicated with arrows). These changes may be, in some respects, an exaggeration of changes that normally occur in adolescence (Rapoport et al., 1999; Thompson et al., 2001a,b).

which myelinate early, showed a more linear pattern of aging than the frontal and parietal neocortices, which continue myelination into adulthood. Posterior temporal cortices, primarily in the left hemisphere, which typically support language functions, have a more protracted course of maturation than any other cortical region.

Another developmental study (Gogtay et al., 2004) created a quantitative time-lapse movie of human cortical development, reconstructed from serial brain MRI scans of children aged 4-21. Dynamic video maps localizing brain changes were derived using high-dimensional elastic deformation mappings to match gyral anatomy across subjects and time. A quadratic statistical model, with random effects, was fitted to the profile of gray matter density against time at each of the 65,536 cortical points (Fig. 7b). The resulting trajectory was animated to create a time-lapse movie (specific frames are shown in c). This revealed a shifting pattern of gray matter loss, appearing first in dorsal parietal and primary sensorimotor regions near the interhemispheric margin, and spreading laterally and caudally into temporal cortices and anteriorly into dorsolateral prefrontal areas. This also supports findings of earlier studies (Giedd et al., 1999; Sowell et al., 1999a,b), with a long-term longitudinal sample. The shifting profile of these changes is observed in a set of video sequences (see URL, http://www.loni.ucla.edu/ thompson/DEVEL/dynamic.html for several of these time-lapse movies).

\section{Statistical maps of brain change}

Statistical brain maps from large populations (Fig. 7) are also likely to help assess how different drug treatments modulate the time course of aging and dementia, and how they interact with developmental maturation of the cortex. In developing dynamic atlases for clinical applications, there is a particular interest in modeling atrophic or developmental processes that speed up or slow down. Diseases may accelerate or their rate of progression may be slowed down by therapy. If individuals are scanned more than twice over large time spans (e.g., Fox et al., 2001; Janke et al., 2001), brain change can be modeled more accurately. To compare dynamic processes in different groups of subjects, nonlinear mixed models can be used (Giedd et al., 1999; Gogtay et al., 2004; Toga and Thompson, 2003a,b) to analyze the registered profiles of brain change. For the $i$ th individual's $j$ th measure we have:

$Y_{i j}=f\left(\operatorname{Age}_{i j}, \underline{\beta}\right)+\varepsilon_{i j}$

Here $Y_{i j}$ signifies the outcome measure at a voxel or surface point, such as growth or tissue loss, $f()$ denotes a constant, linear, quadratic, cubic, or other function of the individual's age for that scan, and $\beta$ denotes the regression/ANOVA coefficients to be estimated. In models whose fit is confirmed as significant, for example, by permutation, loadings on nonlinear parameters may be visualized as attribute maps $\beta(x)$. This reveals the topography of accelerated or decelerated brain change (Fig. 7a; see also Fig. 6). The result is a formal approach to assess whether, and where, brain change is speeding up or slowing down, a key feature in medication studies.

\section{Parameterization of the time axis}

In this statistical model, Age $\left(\mathrm{Age}_{i j}\right)$ may be replaced by time from the onset of disease or medication. This flexibility in parameterizing the time axis allows one to temporally register dynamic patterns using criteria that are expected to bring into line temporal features of interest that appear systematically in a group 


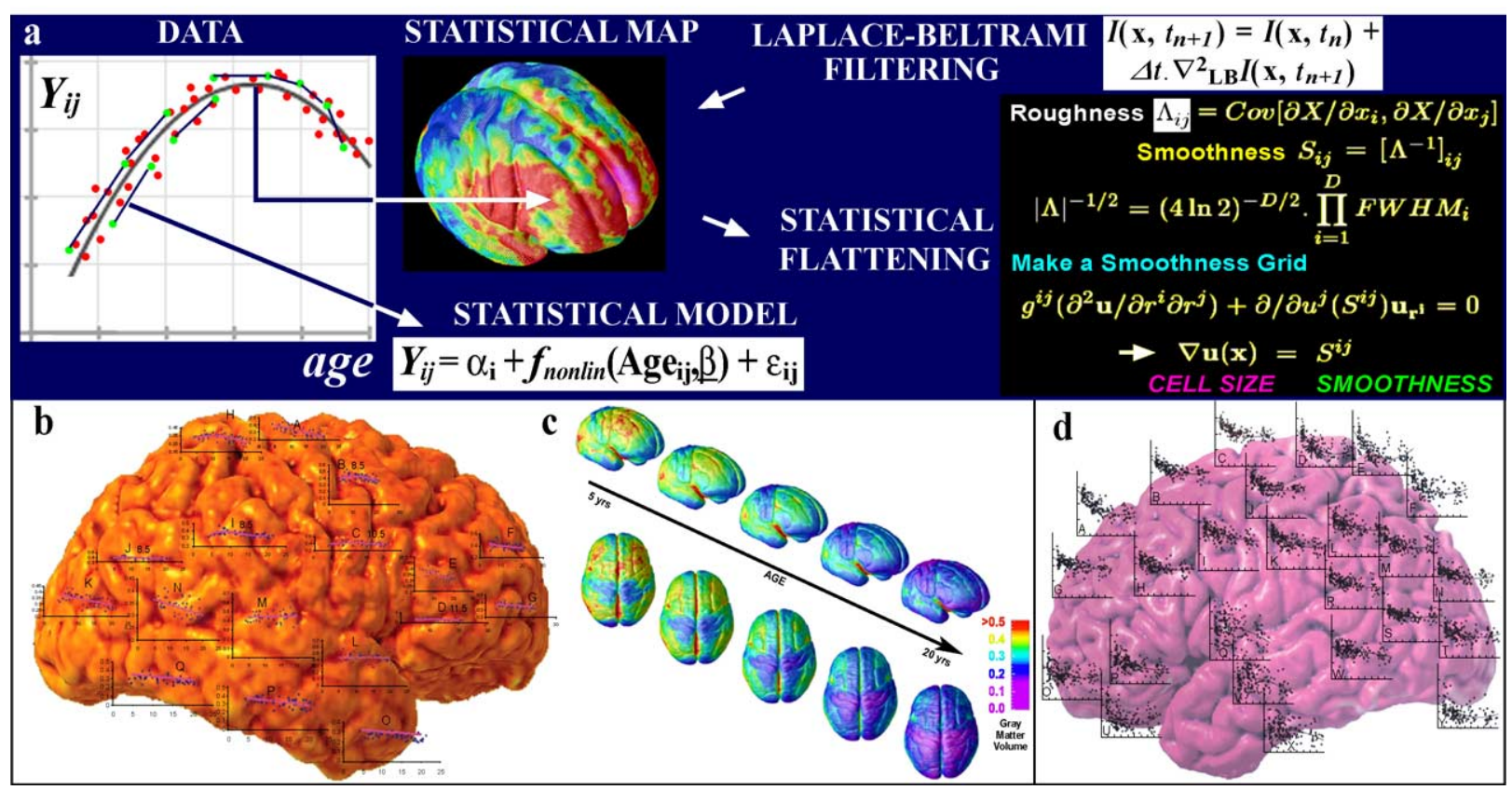

Fig. 7. Mapping brain change over time. The ability to resolve brain changes over time relies on fitting appropriate time-dependent statistical models to data collected from subjects cross sectionally, longitudinally, or both. Nonlinear or mixed statistical models may also be fitted to brain maps collected at different ages to estimate the effects of brain aging or development on the cortex. As illustrated in a, measures $\left(Y_{i j}\right)$ are defined that can be obtained longitudinally (green dots) or once only (red dots) in a group of subjects at different ages. These measures might be gray matter density or cortical thickness, for example. Fitting of statistical models to these data (Statistical Model, lower right) produces estimates of significance values, or statistical parameters such as rates of change, or effects of drug treatment or risk genes. These parameters are then plotted onto the cortex using a color code. Two mathematical tactics (Laplace-Beltrami smoothing and statistical flattening; see main text) can sensitize these analyses to subtle or distributed effects. Panels b and c show the trajectory of cortical gray matter density in 13 children scanned longitudinally every 2 years for 8 years (Gogtay et al., 2004). Panel d shows the trajectory of gray matter loss over the human life span based on a cohort of 176 subjects aged between 7 and 87 (Sowell et al., 2003a,b). [Data reproduced, with permission, from Gogtay et al., Proceedings of the National Academy of Sciences, 2004 (panels b and c), and from Sowell et al., Nature Neuroscience, 2003 (panel d).]

(Janke et al., 2001). For example, the independent variable could be a score from a clinical cognitive measure such as the MiniMental State Examination (MMSE; Janke et al., 2001), which declines over time in AD. Parameterization of dynamic effects using measures other than time (e.g., clinical status or time from disease onset) also provides a mechanism to align new patients' time series with a dynamic atlas (Janke et al., 2001), potentially still further increasing the power to reveal systematic effects. These techniques may hold future benefits in aligning developmental brain data that may be better linked with cognitive or behavioral milestones or underlying hormonal changes than with chronological age per se.

Sensitizing cortical analyses. A fruitful direction for mathematical research lies in sensitizing the statistical models for detecting effects on the cortex using advanced signal processing techniques. Several of these techniques, such as applying filters for feature detection or smoothing, are routine and mathematically wellcharacterized in the Euclidean space of 3D images, but become more complex when applied to data localized on surfaces due to the effects of surface curvature. For example, it is easier to detect cortical signals by smoothing data using covariant filters on the cortical surface, which like the covariant PDEs described earlier account for the curvature and nonuniformities in the surface metric. This technique is under investigation in the computer vision and image restoration literature (Bertalmio et al., 2001; Sapiro, 2001). To increase the detection sensitivity to changes in surface-based data, it is possible to run a time-dependent PDE, such as a Laplace-
Beltrami flow, in the parameter space of the surface. This produces a scale space of diffused data $I(x, t)$ (Chung et al., 2001a,b; Nielsen et al., 1994; Sochen et al., 1998; Worsley, 1996) on the cortex. This acts as a prefilter to enhance detection of effects at different scales (Huiskamp, 1991): $I\left(x, t_{n+1}\right)=I\left(x, t_{n}\right)+\Delta t . \nabla_{\mathrm{LB}}^{2} I\left(x, t_{n+1}\right)$. In this process, the Laplace-Beltrami operator on the cortical manifold can be computed from the divergence of the projected gradient of an implicit function $\psi$, which represents the 3D signed distance to the cortical surface (see Memoli et al., in press, this volume: $\frac{\partial u}{\partial t}=\nabla \cdot\left(\left\|P_{\nabla \psi} \nabla_{u}\right\|\right)$

A second interesting tactic to improve the detection of cortical effects is statistical flattening (Worsley et al., 1999). Most conventional approaches for signal detection (and enhancement) in brain images are based on analytical formulae that describe the distribution of features in random fields (e.g., SPM; Friston et al., 1995). These are difficult to apply if data lie on curved manifolds such as the cortex (Goebel and Singer, 1999; Jones et al., 2000; Taylor and Adler, 2000), as the computed spatial autocorrelation of the surface data (1) depends on the local parameterization (or metric) of the surface, and (2) it may not be stationary (i.e., the same everywhere on the surface). To overcome this and to apply detection formulae for cortical signals that apply to stationary data (i.e., permutation tests on cluster extent or the Euler characteristics of suprathreshold statistics), a computational grid can be adapted to the roughness tensor of the data using a datadriven PDE (a process called statistical flattening; Worsley et al., 1999). This can be thought of as a smooth change of coordinates on the surface; for example, instead of using the conformal 
parameterization of the cortex described earlier, we run a partial differential equation:

$g^{i j}\left(\partial^{2} u / \partial r^{i} \partial r^{j}\right)+\partial / \partial u^{j}\left(S^{i j}\right) u_{r} i=0$,

in the parameter space of the group average cortex. This generates a deformed grid $u(r)$ whose deformation gradient tensor approximates the smoothness tensor $S^{i j}$ of the normalized residuals of the data on the surface (here $g^{i j}$ is the contravariant metric tensor of the grid). If the smoothness tensor has non-zero curvature (and is therefore not realizable as a deformation tensor), the deformation gradient approximates it in the Frobenius matrix norm. Relative to this new computational grid, the residuals become stationary and isotropic, and $P$ values for the gray matter reductions, or changes in other cortical features, can be evaluated more powerfully. Permutation tests can then count statistics of suprathreshold cluster size relative to this new computational grid, eliminating the nonstationary smoothness that degrades the power of tests that measure area and extent of suprathreshold statistics on the cortical surface.

\section{Conclusion}

As a whole, the structural brain mapping methods described here differ substantially from conventional volumetric measures of anatomy. Brain mapping algorithms can employ geometric models derived from MRI data to create spatially detailed maps of brain changes. In longitudinal studies, these allow visualization of rates and profiles of tissue growth and loss. Computational atlases can store these maps from large patient cohorts, including those at genetic risk. They can uncover nonlinear brain changes over the human life span and chart the path of cortical change in development and dementia. There are two urgent applications for these computational methods. The first is to map therapeutic effects in drug trials that aim to slow disease progression or stabilize or reverse developmental deficits. Dynamic maps have potential as a surrogate outcome measure for disease stabilization therapies, providing regional anatomic localization of any beneficial effects. Any improvement in the set of neuroimaging biomarkers will make it considerably easier to monitor the proactive benefits of treatment before diseases progress irremediably. Second, there is interest in developing neuroimaging markers that are linked with genetic variation (Cannon et al., 2002; Thompson et al., 2001a,b). This is likely to shed light on the transmission of heritable disease and heritable aspects of cognition. With the rapid pace of developments in both fields, the merger of computational methods from genetics and from imaging is likely to engender a second revolution in our understanding of the human brain.

\section{Acknowledgments}

This work was funded by grants from the National Institute for Biomedical Imaging and Bioengineering, the National Center for Research Resources, and the National Institute on Aging (to P.T.: R21 EB01651, R21 RR019771, P50 AG016570), by the National Institute of Mental Health, the National Institute of Drug Abuse, and the March of Dimes, (to E.R.S.: K01 MH01733, R21 DA15878, MOD 5FY03-12), by GlaxoSmithKline Pharmaceuticals UK, and by a Human Brain Project grant to the International Consortium for Brain Mapping, funded jointly by NIMH and
NIDA (P20 MH/DA52176; P41 RR13642, and P20 MH65166 to A.W.T.). Additional support was provided by NIMH intramural funding (J.L.R.), by NIMH grant R01 MH52857 (T.D.C.), and by a grant to the FIRST Biomedical Informatics Research Network (BIRN, http:/www.nbirn.net), which is funded by the NCRR and NIH (RR00827).

\section{References}

Alouges, F., Ghidaglia, J.M., 1997. Minimizing the Oseen-Frank energy for nematic liquid crystals: algorithms and numerical results. Ann. I.H.P., Phys. Theor. 66, 411-447.

Angenent, S., Haker, S., Tannenbaum, A., Kikinis, R., 1999. On the Laplace-Beltrami operator and brain surface flattening. IEEE Trans. Med. Imag. 18 (8), 700-711.

Annese, J., Pitiot, A., Toga, A.W., 2002. 3D cortical thickness maps from histological volumes. NeuroImage 13 (6), S858 (Part 2).

Ashburner, J., Friston, K.J., 2000. Voxel-based morphometry-The methods. Neuroimage 11 (6), 805-821.

Ashburner, J., Hutton, C., Frackowiak, R., Johnsrude, I., Price, C., Friston, K., 1998. Identifying global anatomical differences: deformation-based morphometry. Hum. Brain Mapp. 6 (5-6), 348-357.

Ashburner, J., Csernansky, J.G., Davatzikos, C., Fox, N.C., Frisoni, G.B., Thompson, P.M., 2003. Computer-assisted imaging to assess brain structure in healthy and diseased brains. Lancet Neurology 2, $78-88$.

Avants, B., Gee, J.C., 2004. Geodesic estimation for large deformation anatomical shape averaging and interpolation. In: Thompson, P.M., Miller, M.I., Ratnanather, J.T., Poldrack, R., Nichols, T.E. (Eds.), NeuroImage, Special Issue on Mathematics Brain Imaging. This issue.

Bakircioglu, M., Joshi, S., Miller, M.I., 1999. Landmark matching on brain surfaces via large deformation diffeomorphisms on the sphere. Proc. SPIE Medical Imaging.

Ballmeier, M., Sowell, E.R., Thompson, P.M., Kumar, A., Lavretsky, H., Wellcome, S.E., DeLuca, H., Toga, A.W., 2003. Mapping brain size and cortical surface gray matter changes in elderly depression. Biol. Psychiatry 55 (4), 382-389 (2004 Feb 15).

Bankman, I.N., 1999. Handbook of Medical Imaging: Processing and Analysis. Academic Press.

Bertalmio, M., Bertozzi, A.L., Sapiro, G., 2001. Navier-Stokes, fluid dynamics, and image and video imprinting. Proc. - IEEE Comput. Conf. Vis. Pattern Recognit. December, Hawaii.

Blanton, R.E., Levitt, J.L., Thompson, P.M., Capetillo-Cunliffe, L.F., Sadoun, T., Williams, T., McCracken, J.T., Toga, A.W., 2001. Mapping cortical variability and complexity patterns in the developing human brain. Psychiatry Res.: Neuroimaging 107, 29-43.

Bookstein, F., 2001. Voxel-based morphometry should not be used with imperfectly registered images. NeuroImage 14, 1454-1462.

Braak, H., Del Tredici, K., Schultz, C., Braak, E., 2000. Vulnerability of select neuronal types to Alzheimer's disease. Ann. N. Y. Acad. Sci. 924, 53-61.

Cachier, P., Ayache, N., 2004. Isotropic energies, filters and splines for vectorial regularization. J. Math. Imaging Vis. 20 (3), 251-265.

Cannon, T.D., Thompson, P.M., van Erp, T., Toga, A.W., Poutanen, V.-P., Huttunen, M., Lönnqvist, J., Standertskjöld-Nordenstam, C.-G., Narr, K.L., Khaledy, M., Zoumalan, C.I., Dail, R., Kaprio, J., 2002. Cortex mapping reveals heteromodal gray matter deficits in monozygotic twins discordant for schizophrenia. Proc. Natl. Acad. Sci. U. S. A. 99 (5), $3228-3233$.

Cao, J., Worsley, K.J., 1999. The geometry of the Hotelling's T-squared random field with applications to the detection of shape changes. Ann. Stat. 27, 925-942.

Christensen, G.E., Rabbitt, R.D., Miller, M.I., 1996. Deformable templates using large deformation kinematics. IEEE Trans. Image Process. 5 (10), 1435-1447 (Oct). 
Chung, M.K., 2001. Statistical Morphometry in Neuroanatomy, Montreal: $\mathrm{PhD}$ Thesis, McGill University.

Chung, M.K., Worsley, K.J., Taylor, J., Ramsay, J.O., Robbins, S., Evans, A.C., 2001. Diffusion smoothing on the cortical surface. NeuroImage 13S, 95.

Chung, M.K., Worsley, K.J., Paus, T., Cherif, C., Giedd, J.N., Rapoport, J.L., Evans, A.C., 2001. A unified statistical approach to deformationbased morphometry. NeuroImage 14, 595-606.

Csernansky, J.G., Joshi, S., Wang, L., et al., 1998. Hippocampal morphometry in schizophrenia by high dimensional brain mapping. Proc. Natl. Acad. Sci. U. S. A. 95 (19), 11406-11411.

Davatzikos, C., 1996. Spatial normalization of 3D brain images using deformable models. J. Comput. Assist. Tomogr. 20 (4), 656-665 (Jul.-Aug)

Davatzikos, C., Genc, A., Xu, D., Resnick, S.M., 2001. Voxel-based morphometry using the RAVENS maps: methods and validation using simulated longitudinal atrophy. NeuroImage 14 (6), 1361-1369 (Dec).

Davis, M.H., Khotanzad, A., Flamig, D.P., 1997. 3D image matching using a radial basis function neural network. Proc. 1996 World Congress on Neural Networks, San Diego, California, Sept. 15-18. pp. $1174-1179$.

Drury, H.A., Van Essen, D.C., 1997. Functional specializations in human cerebral cortex analyzed using the Visible Man surface-based atlas. Hum. Brain Mapp. 5, 233-237.

Dupuis, P., Grenander, U., Miller, M.I., 1998. Variational problems on flows of diffeomorphisms for image matching. Q. Appl. Math 56 (3), 587-600 (Sep).

Edgington, E.S., 1969. Statistical Inference: The Distribution-Free Approach. McGraw-Hill, New York.

Edgington, E.S., 1995. Randomisation Tests. Marcel Dekker, New York.

Einstein, A., 1914. Covariance properties of the field equations of the theory of gravitation based on the generalized theory of relativity. Z. Angew. Math. Phys. 63, 215-225.

Evans, A.C., Collins, D.L., Neelin, P., MacDonald, D., Kamber, M., Marrett, T.S., 2000. Three-dimensional correlative imaging: applications in human brain mapping. In: Thatcher, R.W., Hallett, M., Zeffiro, T., John, E.R., Huerta, M. (Eds.), Functional Neuroimaging: Technical Foundations. pp. $145-162$.

Fischl, B., Dale, A.M., 2000. Measuring the thickness of the human cerebral cortex from magnetic resonance images. Proc. Natl. Acad. Sci. U. S. A. 97 (20), 11050-11055.

Fischl, B., Sereno, M.I., Tootell, R.B.H., Dale, A.M., 1999. High-resolution inter-subject averaging and a coordinate system for the cortical surface. Hum. Brain Mapp. 8 (4), 272-284.

Fischl, B., Liu, A., Dale, A.M., 2001. Automated manifold surgery: constructing geometrically accurate and topologically correct models of the human cerebral cortex. IEEE Trans. Med. Imag. 20 (1), 70-80.

Fitzpatrick, J.M., Sonka, M., 2000. Medical Image Processing and Analysis. SPIE Press.

Fox, N.C., Crum, W.R., Scahill, R.I., Stevens, J.M., Janssen, J.C., Rossor, M.N., 2001. Imaging of onset and progression of Alzheimer's disease with voxel-compression mapping of serial magnetic resonance images. Lancet 358 (9277), 201-205.

Friston, K.J., Holmes, A.P., Worsley, K.J., Poline, J.P., Frith, C.D., Frackowiak, R., 1995. Statistical parametric maps in functional imaging: a general linear approach. Hum. Brain Mapp. 2, 189-210.

Gee, J.C., Bajcsy, R.K., 1998. Elastic matching: continuum-mechanical and probabilistic analysis. In: Toga, A.W. (Ed.), Brain Warping. Academic Press, San Diego.

Gerig, G., Styner, M., Weinberger, D.R., Jones, D., Lieberman, J.A., 2001. Shape Analysis of Brain Ventricles using SPHARM. IEEE Workshop on Mathematical Methods in Biomedical Image Analysis.

Giedd, J.N., Blumenthal, J., Jeffries, N.O., Castellanos, F.X., Liu, H., Zijdenbos, A., Paus, T., Evans, A.C., Rapoport, J.L., 1999. Brain development during childhood and adolescence: a longitudinal MRI study. Nat. Neurosci. 2 (10), 861-863 (Oct).
Goebel, R., Singer, W., 1999. Cortical surface-based statistical analysis of functional activation. NeuroImage 98, 64.

Gogtay, N., Giedd, J.N., Lusk, L., Hayashi, K.M., Greenstein, D., Vaitiuzis, C., Nugent, T.F., Herman, D.H., Classen, L., Toga, A.W., Rapoport, J.L., Thompson, P.M., 2004. Dynamic mapping of human cortical development during childhood and adolescence. Proc. Natl. Acad. Sci. 101 (21), 8174-8179 (May 25).

Good, C.D., Johnsrude, I.S., Ashburner, J., Henson, R.N., Friston, K.J., Frackowiak, R.S.J., 2001. A voxel-based morphometric study of ageing in 465 normal adult human brains. NeuroImage 14 (1 Pt. 1), 21-36 (Jul).

Gray, J.R., Thompson, P.M., 2004. Neurobiology of intelligence: science and ethics. Nat. Rev., Neurosci. 5 (6), 471-482 (Jun).

Grenander, U., Miller, M.I., 1998. Computational Anatomy: An Emerging Discipline. Technical Report, Dept. of Mathematics, Brown University.

Gu, X., Wang, Y.L., Chan, T., Thompson, P.M., Yau, S.T., 2003. Genus zero conformal mapping and its application to brain surface mapping. In: Taylor, C.J., Noble, J.A. (Eds.), 18th International Conference on Information Processing in Medical Imaging (IPMI2003), Lect. Notes Comput. Sci., vol. 2732. Springer-Verlag, pp. 172-184.

Haker, S., Angenent, S., Tannenbaum, A., Kikinis, R., Sapiro, G., Halle, M., 2000. Conformal surface parameterization for texture mapping. IEEE Transactions on Visualization and Computer Graphics, pp. 1-9.

Huiskamp, G., 1991. Difference formulas for the surface Laplacian on a triangulated surface. J. Comput. Phys. 95, 477-496.

Hurdal, M.K., Stephenson, K., 1991. Cortical cartography using the discrete conformal approach of circle packings. In: Thompson, P.M., Miller, M.I., Ratnanather, J.T., Poldrack, R., Nichols, T.E. (Eds.), Neuroimage, Special Issue on Mathematics in Brain Imaging. In Press.

Jack, C.R., Slomkowski, M., Gracon, S., et al., 2003. MRI as a biomarker of disease progression in a therapeutic trial of milameline for $\mathrm{AD}$. Neurology 60, 253-260.

Janke, A.L., de Zubicaray, G., Rose, S.E., Griffin, M., Chalk, J.B., Galloway, G.J., 2001. 4D deformation modeling of cortical disease progression in Alzheimer's dementia. Magn. Reson. Med. 46 (4), 661-666.

Jernigan, T., Salmon, D.P., Butters, N., Hesselink, J.R., 1991. Cerebral structure on MRI, part II: specific changes in Alzheimer's and Huntington's diseases. Biol. Psychiatry 29, 68-81.

Jernigan, T.L., Archibald, S.L., Fennema-Notestine, C., Gamst, A.C., Stout, J.C., Bonner, J., Hesselink, J.R., 2001. Effects of age on tissues and regions of the cerebrum and cerebellum. Neurobiol. Aging 22 (4), 581-594 (Jul-Aug).

Jones, S.E., Buchbinder, B.R., Aharon, I., 2000. Three-dimensional mapping of cortical thickness using Laplace's equation. Hum. Brain Mapp. 11 (1), 12-32.

Joshi, S.C., Miller, M.I., Christensen, G.E., Banerjee, A., Coogan, T.A., Grenander, U., 1995. Hierarchical brain mapping via a generalized Dirichlet solution for mapping brain manifolds, Vision Geometry IV. Conference on Optical Science, Engineering and Instrumentation. San Diego, CA, pp. 278-289.

Joshi, S., Miller, M.I., Grenander, U., 1998. On the geometry and shape of brain sub-manifolds. Int. J. Patt. Recogn. Artif. Intell.

Joshi, A.A., Leahy, R.M., Thompson, P.M., Shattuck, D.W., 1998. Cortical surface parameterization by P-harmonic energy minimization. In: Leahy, R., Roux, C. (Eds.), IEEE International Symposium on Biomedical Imaging. Arlington, VA.

Kennedy, D.N., Lange, N., Makris, N., Bates, J., Meyer, J., Caviness Jr., V.S., 1998. Gyri of the human neocortex: an MRI-based analysis of volume and variance. Cereb. Cortex 8 (4), 372-384.

Kindlmann, G.L., Weinstein, D.M., Lee, A.D., Toga, A.W., Thompson, P.M., 1998. Visualization of anatomic covariance tensor fields. Proc. IEEE Engineering in Medicine and Biology Society (EMBS), San Francisco, CA. pp. 1-5.

Kruggel, F., Bruckner, M.K., Arendt, T., Wiggins, C.J., von Cramon, D.Y., 2001. Analyzing the neocortical fine structure. Proc. Info. Process. in Med. Imaging. Springer Press, pp. 239-245. 
Lancaster, J.L., Kochunov, P.V., Thompson, P.M., Toga, A.W., Fox, P.T., 2003. Asymmetry of the brain surface from deformation field analysis. Hum. Brain Mapp. 19 (2), $79-89$ (June).

Leahy, R.M., Insana, M., 2001. Information Processing in Medical Imaging. UC Davis. June.

Leow, A.D., Huang, S.C., Osher, S., Toga, A.W., Thompson, P.M., 2004. Nonlinear registration using non-parametric geometric features. IEEE Trans. Image Process (in press).

Lin, J.J., Salamon, N., Lee, A.D., Dutton, R.A., Geaga, J.A., Hayashi, K.M., London, E.D., Toga, A.W., Engel, J., Thompson, P.M., 2004. Mapping of neocortical gray matter loss in patients with mesial temporal lobe epilepsy with hippocampal sclerosis. Proc. 34th Annual Conference of the Society for Neuroscience, San Diego, CA, Oct. 23-27.

Liseikin, V.D., 1991. On a variational method for generating adaptive grids on N-dimensional surfaces, Doklady Akademii Nauk. CCCP 319 (3), $546-549$.

Luders, E., Narr, K.L., Thompson, P.M., Rex, D.E., Jancke, L., Toga, A.W., 2004. Gender differences in cortical complexity. Nat. Neurosci. (in press).

MacDonald, D., 1998. A Method for Identifying Geometrically Simple Surfaces from Three Dimensional Images. PhD thesis, McGill Univ., Canada.

Mangin, J.F., Rivière, D., Cachia, A., Duchesnay, E., Cointepas, Y., Papadopoulos-Orfanos, D., Scifo, P., Ochiai, T., Régis, J., 2004. In Thompson, P.M., Miller, M.I., Ratnanather, J.T., Poldrack, R., Nichols, T.E. (Eds.), A framework to study the cortical folding patterns, Neuroimage, Special Issue on Mathematics in Brain Imaging. In Press.

Mazziotta, J.C., Toga, A.W., Evans, A.C., Fox, P.T., Lancaster, J., Zilles, K., Woods, R.P., Paus, T., Simpson, G., Pike, B., Holmes, C.J., Collins, D.L., Thompson, P.M., MacDonald, D., Schormann, T., Amunts, K., Palomero-Gallagher, N., Parsons, L., Narr, K.L., Kabani, N., Le Goualher, G., Boomsma, D., Cannon, T., Kawashima, R., Mazoyer, B., 2001. A probabilistic atlas and reference system for the human brain [Invited Paper]. J. R. Soc. 356 (1412), 1293-1322 (29th August 2001).

Memoli, F., Sapiro, G., Osher, S., 2002. Solving Variational Problems and Partial Differential Equations Mapping into General Target Manifolds. University of Minnesota IMA TR. January, available at www.ima. umn.edu.

Memoli, F., Sapiro, G., Thompson, P.M., 2002. Implicit brain imaging. In Thompson, P.M., Miller, M.I., Ratnanather, J.T., Poldrack, R., Nichols, T.E. (Eds.), NeuroImage, Special Issue on Mathematics in Brain Imaging. In Press.

Mesulam, M.M., 2000. A plasticity-based theory of the pathogenesis of Alzheimer's disease. Ann. N. Y. Acad. Sci. 924, 42-52.

Miller, M.I., 2000. Computational anatomy: shape, growth and atrophy comparison via diffeomorphisms. In: Thompson, P.M., Miller, M.I., Ratnanather, J.T., Poldrack, R., Nichols, T.E. (Eds.), NeuroImage, Special Issue on Mathematics in Brain Imaging. In Press.

Miller, M.I., Massie, A.B., Ratnanather, J.T., Botteron, K.N., Csernansky, J.G., 2000. Bayesian construction of geometrically based cortical thickness metrics. NeuroImage 12 (6), 676-687.

Miller, M.I., Trouve, A., Younes, L., 2002. On the metrics and EulerLagrange equations of computational anatomy. Annu. Rev. Biomed. Eng. 4, 375-405.

Narr, K.L., Cannon, T.D., Woods, R.P., Thompson, P.M., Kim, S., Asunction, D., van Erp, T.G., Poutanen, V.P., Huttunen, M., Lonnqvist, J., Standerksjold-Nordenstam, C.G., Kaprio, J., Mazziotta, J.C., Toga, A.W., 2002. Genetic contributions to altered callosal morphology in schizophrenia. J. Neurosci. 22 (9), 3720-3729 (May 1).

Narr, K.L., Bilder, R.M., Kim, S., Thompson, P.M., Szeszko, P., Robinson, D., Luders, E., Toga, A.W., 2004a. Abnormal gyral complexity in first episode schizophrenia. Biol. Psychiatry 55 (8), 859-867 (15 Apr).

Narr, K.L., Bilder, R.M., Woods, R.P., Rex, D.E., Szeszko, P., Robinson, D., Wang, Y.P., DeLuca, H., Toga, A.W., Thompson, P.M., 2004b. Mapping Cortical Thickness and Gray Matter Density in First Episode Schizophrenia. International Conference of the Organization for Human Brain Mapping, Budapest, Hungary. June 13-17.
Nichols, T.E., Holmes, A.P., 2002. Nonparametric permutation tests for functional neuroimaging: a primer with examples. Hum. Brain Mapp. $15(1), 1-25$

Nielsen, M., Florack, L., Deriche, R., 1994. Regularization and scale space: INRIA Technical Report. 1994 September

Osher, S.J., Sethian, J.A., 1988. Fronts propagation with curvature dependent speed: algorithms based on Hamilton-Jacobi formulations. J. Comput. Phys. 79, 12-49.

Paus, T., Zijdenbos, A., Worsley, K., Collins, D.L., Blumenthal, J., Giedd, J.N., Rapoport, J.L., Evans, A.C., 1999. Structural maturation of neural pathways in children and adolescents: in vivo study. Science 283 (5409), 1908-1911 (Mar 19).

Polyakov, A.M., 1987. Gauge Fields and Strings. Harwood, New York

Posthuma, D., De Geus, E.J., Baare, W.F., Hulshoff Pol, H.E., Kahn, R.S., Boomsma, D.I., 2002. The association between brain volume and intelligence is of genetic origin. Nat. Neurosci. 5 (2), 83-84 (Feb).

Rapoport, J.L., Giedd, J.N., Blumenthal, J., Hamburger, S., Jeffries, N., Fernandez, T., Nicolson, R., Bedwell, J., Lenane, M., Zijdenbos, A., Paus, T., Evans, A., 1999. Progressive cortical change during adolescence in childhood-onset schizophrenia. A longitudinal magnetic resonance imaging study. Arch. Gen. Psychiatry 56 (7), 649-654 (Jul).

Rasser, P.E., Johnston, P.J., Lagopoulos, J., Ward, P.B., Schall, U., Thienel, R., Bender, S., Toga, A.W., Thompson, P.M., 2004. Analysis of fMRI BOLD activation during the tower of London task using gyral pattern and intensity averaging models of cerebral cortex. NeuroImage (in press).

Sapiro, G., 2001. Geometric Partial Differential Equations and Image Processing. Cambridge Univ. Press.

Sereno, M.I., Dale, A.M., Liu, A., Tootell, R.B., 1996. A surface-based coordinate system for a canonical cortex. 2nd International Conference Human Brain Mapping. NeuroImage, Boston, MA, pp. S252.

Sochen, N., Kimmel, R., Malladi, R., 1998. A general framework for low level vision. IEEE Trans. Image Process. 7, 310-318.

Sowell, E.R., Thompson, P.M., Holmes, C.J., Jernigan, T.L., Toga, A.W., 1999a. In vivo evidence for post-adolescent brain maturation in frontal and striatal regions. Nat. Neurosci. 2 (10), 859-861.

Sowell, E.R., Thompson, P.M., Holmes, C.J., Batth, R., Jernigan, T.L., Toga, A.W., 1999b. Localizing age-related changes in brain structure between childhood and adolescence using statistical parametric mapping. NeuroImage 9, 587-597.

Sowell, E.R., Thompson, P.M., Tessner, K.D., Toga, A.W., 2001. Mapping continued brain growth and gray matter density reduction in dorsal frontal cortex: inverse relationships during postadolescent brain maturation. J. Neurosci. 21, 8819-8829.

Sowell, E.R., Thompson, P.M., Peterson, B.S., Mattson, S.N., Welcome, S.E., Henkenius, A.L., Riley, E.P., Jernigan, T.L., Toga, A.W., 2002. Mapping cortical gray matter asymmetry patterns in adolescents with heavy prenatal alcohol exposure. NeuroImage 17 (4), 1807-1819 (Dec).

Sowell, E.R., Thompson, P.M., Rex, D.E., Kornsand, D.S., Jernigan, T.L., Toga, A.W., 2002a. Mapping sulcal pattern asymmetry and local cortical surface gray matter distribution in vivo: maturation in perisylvian cortices. Cereb. Cortex 12 (1), 17-26 (Jan)

Sowell, E.R., Thompson, P.M., Mattson, S.N., Tessner, K.D., Jernigan, T.L., Riley, E.P., Toga, A.W., 2002b. Regional brain shape abnormalities persist into adolescence after heavy prenatal alcohol exposure. Cereb. Cortex 12, 856-865.

Sowell, E.R., Peterson, B.S., Thompson, P.M., Welcome, S.E., Henkenius, A.L., Toga, A.W., 2003a. Mapping cortical change across the human lifespan. Nat. Neurosci. 6 (3), 309-315 (March).

Sowell, E.R., Thompson, P.M., Welcome, S.E., Henkenius, A.L., Toga, A.W., Peterson, B.S., 2003b. Cortical abnormalities in children and adolescents with attention-deficit hyperactivity disorder. Lancet 22 (362 (9397)), 1699-1707 (December).

Sowell, E.R., Thompson, P.M., Leonard, C.M., Welcome, S.E., Kan, E., Toga, A.W., 2004a. Longitudinal Mapping of Cortical Thickness and Brain Growth in Normal Children. J. Neuroscience (in press). 
Sowell, E.R., Thompson, P.M., Yoshii, J., Kan, E., Toga, A.W., Peterson, B.S., 2004b. Gray matter thickness abnormalities mapped in children with Tourette syndrome. Proc. 34th Annual Conference of the Society for Neuroscience, San Diego, CA. Oct. 23-27.

Taylor, J.E., Adler, R.J., 2000. Euler characteristics for Gaussian fields on manifolds. Ann. Probab.

Thirion, J.-P., Prima, S., Subsol, S., 2000. Statistical analysis of dissymmetry in volumetric medical images. Med. Image Anal. 4 (2), 111-121.

Thompson, P.M., Toga, A.W., 1996. A surface-based technique for warping 3-dimensional images of the brain. IEEE Trans. Med. Imag. 15 (4), 1-16 (August).

Thompson, P.M., Toga, A.W., 2002. A framework for computational anatomy [Invited Paper]. Comput. Vis. Sci. 5, 1-12.

Thompson, P.M., Toga, A.W., 2003a. Alzheimer's Disease: MRI imaging of progressive brain change [Review Article]. In: Adelman, G., Smith, B.H. (Eds.), Encyclopedia of Neuroscience. Elsevier, Amsterdam. Nov. 2003

Thompson, P.M., Toga, A.W., 2003b. Cortical diseases and cortical localization [Review Article]. Nature Encyclopedia of the Life Sciences (CD-ROM). Macmillan Press, London.

Thompson, P.M., Schwartz, C., Lin, R.T., Khan, A.A., Toga, A.W., 1996a. 3D statistical analysis of sulcal variability in the human brain. J. Neurosci. 16 (13), 4261-4274 (July).

Thompson, P.M., Schwartz, C., Toga, A.W., 1996b. High-resolution random mesh algorithms for creating a probabilistic 3D surface atlas of the human brain. NeuroImage 3 (1), 19-34 (March).

Thompson, P.M., MacDonald, D., Mega, M.S., Holmes, C.J., Evans, A.C., Toga, A.W., 1997. Detection and mapping of abnormal brain structure with a probabilistic atlas of cortical surfaces. J. Comput. Assist. Tomogr. 21 (4), 567-581 (Jul.-Aug.).

Thompson, P.M., Moussai, J., Khan, A.A., Zohoori, S., Goldkorn, A., Mega, M.S., Small, G.W., Cummings, J.L., Toga, A.W., 1998. Cortical variability and asymmetry in normal aging and Alzheimer's Disease. Cereb. Cortex 8 (6), 492-509 (Sept).

Thompson, P.M., Giedd, J.N., Woods, R.P., MacDonald, D., Evans, A.C., Toga, A.W., 2000a. Growth patterns in the developing brain detected by using continuum-mechanical tensor maps. Nature 404 (6774), 190-193 (March 9).

Thompson, P.M., Woods, R.P., Mega, M.S., Toga, A.W., 2000b. Mathematical/computational challenges in creating population-based brain atlases, [Invited Paper]. Hum. Brain Mapp. 9 (2), 81-92 (Feb).

Thompson, P.M., Cannon, T.D., Narr, K.L., van Erp, T., Khaledy, M., Poutanen, V.-P., Huttunen, M., Lönnqvist, J., Standertskjöld-Nordenstam, C.-G., Kaprio, J., Dail, R., Zoumalan, C.I., Toga, A.W., 2001a. Genetic Influences on brain structure. Nat. Neurosci. 4 (12), 1253-1258 (Dec).

Thompson, P.M., Vidal, C.N., Giedd, J.N., Gochman, P., Blumenthal, J., Nicolson, R., Toga, A.W., Rapoport, J.L., 2001b. Mapping adolescent brain change reveals dynamic wave of accelerated gray matter loss in very early-onset schizophrenia. Proc. Natl. Acad. Sci. U. S. A. 98 (20), 11650-11655 (September 25).

Thompson, P.M., Hayashi, K.M., de Zubicaray, G., Janke, A.L., Rose, S.E., Semple, J., Herman, D., Hong, M.S., Dittmer, S., Doddrell, D.M., Toga, A.W., 2003. Dynamics of gray matter loss in Alzheimer's disease. J. Neurosci. 23 (3), 994-1005 (Feb. 1).

Thompson, P.M., Hayashi, K.M., de Zubicaray, G., Janke, A.L., Rose, S.E., Semple, J., Hong, M.S., Herman, D., Gravano, D., Doddrell,
D.M., Toga, A.W., 2004a. Mapping hippocampal and ventricular change in Alzheimer's disease. NeuroImage 22 (4), 1754-1766.

Thompson, P.M., Hayashi, K.M., Simon, S., Geaga, J., Hong, M.S., Sui, Y., Lee, J.Y., Toga, A.W., Ling, W.L., London, E.D., 2004b. Structural abnormalities in the brains of human subjects who use methamphetamine. J. Neurosci., 14, 6028-6036.

Thompson, P.M., Lee, A.D., Dutton, R.A., Geaga, J.A., Hayashi, K.M., Bacheller, J.D., Eckert, M.A., Toga, A.W., Reiss, A.L., 2004c. Fractal complexity of the human cortex is increased in Williams syndrome. International Conference of the Organization for Human Brain Mapping, Budapest, Hungary. (June 13-17, 2004).

Toga, A.W., 1998. Brain Warping. Academic Press, New York.

Toga, A.W., Mazziotta, J.C., 2002. Brain Mapping: The Methods, (second revised ed.). Academic Press.

Toga, A.W., Thompson, P.M., 2003a. Mapping brain asymmetry [Invited Paper]. Nat. Rev. Neurosci. 4 (1), 37-48 (January)

Toga, A.W., Thompson, P.M., 2003b. Temporal dynamics of brain anatomy [Invited Paper]. Annu. Rev. Biomed. Eng. 5, 119-145 (Aug.).

Tosun, D., Rettmann, M.E., Han, X., Tao, X., Xu, C., Resnick, S.M., Pham, D., Prince, J.L., 2004. In: Thompson, P.M., Miller, M.I., Ratnanather, J.T., Poldrack, R., Nichols, T.E. (Eds.), Cortical surface segmentation and mapping, Neuroimage, Special Issue on Mathematics in Brain Imaging. In Press.

Van Erp, T.G.M., Thompson, P.M., Kieseppa, T., Tran, H.L., Correll, C.M., Hayashi, K.M., Haukka, J., Paronen, T., Poutanen, V.P., Kaprio, J., Lonnqvist, J., Toga, A.W., Cannon, T.D., 2004. Brain Morphology in Twins Discordant for Bipolar Disorder. 24th Conference of the International Society for Neuropsychopharmacology (CINP), Paris, France. June 20-24, 2004.

van Essen, D.C., 2003. In: Thompson, P.M., Miller, M.I., Ratnanather, J.T., Poldrack, R., Nichols, T.E. (Eds.), Surface-based approaches to spatial localization and registration in primate cerebral cortex, Neuroimage, Special Issue on Mathematics in Brain Imaging. In Press.

Wang, Y.L., Gu, X., Chan, T., Thompson, P.M., Yau, S.T., 2004. Genus zero conformal mapping and its application to brain surface mapping. IEEE Trans. Med. Imag. 23 (8), 949-958.

Woods, R.P., 2003. Characterizing volume and surface deformations in an atlas framework: Theory, applications, and implementation. Neuroimage 18, 769-788.

Worsley, K.J., 1996. Searching scale space for activation in PET images. Hum Brain Mapp. 4, 74-90.

Worsley, K.J., Andermann, M., Koulis, T., MacDonald, D., Evans, A.C., 1999. Detecting changes in nonisotropic images. Hum. Brain Mapp. 8 (2-3), 98-101.

Wright, I.C., McGuire, P.K., Poline, J.B., Travere, J.M., Murray, R.M., Frith, C.D., Frackowiak, R.S.J., Friston, K.J., 1995. A voxel-based method for the statistical analysis of gray and white matter density applied to schizophrenia. NeuroImage 2, 244-252.

Yezzi, A., Prince, J., 2001. A PDE Approach for Measuring Tissue Thickness. CVPR. Kauai.

Zeineh, M.M., Engel, S.A., Thompson, P.M., Bookheimer, S., 2003. Dynamics of the hippocampus during encoding and retrieval of facename pairs. Science 299 (5606), 577-580 (January 24).

Zijdenbos, A., Evans, A., Riahi, F., Sled, J., Chui, H.-C., Kollokian, V., . Automatic quantification of multiple sclerosis lesion volume using stereotactic space. Proc. 4th Vis. Biomed. Comp. (VBC), Hamburg, Germany. 